\title{
First ALMA Light Curve Constrains Refreshed Reverse Shocks and Jet Magnetization in GRB 161219B
}

Tanmoy Laskar ${ }^{1,2,16}$ (D), Kate D. Alexander ${ }^{3}$ (D), Edo Berger ${ }^{3}$ (D) , Cristiano Guidorzi $^{4}$ (D), Raffaella Margutti ${ }^{5}$ (D), Wen-fai Fong ${ }^{5,17}$ (iD), Charles D. Kilpatrick ${ }^{6}$ (D), Peter Milne ${ }^{7}$ (D), Maria R. Drout ${ }^{8,18}$, C. G. Mundell ${ }^{9}$, Shiho Kobayashi ${ }^{10}$ (D), Ragnhild Lunnan ${ }^{11,12}$ (D), Rodolfo Barniol Duran ${ }^{13}$, Karl M. Menten ${ }^{14}$ (D) , Kunihito Ioka $^{15}$ (D), and Peter K. G. Williams ${ }^{3}$ (D)

${ }^{1}$ National Radio Astronomy Observatory, 520 Edgemont Road, Charlottesville, VA 22903, USA

${ }^{2}$ Department of Astronomy, University of California, 501 Campbell Hall, Berkeley, CA 94720-3411, USA

${ }_{3}^{3}$ Department of Astronomy, Harvard University, 60 Garden Street, Cambridge, MA 02138, USA

${ }^{4}$ Department of Physics and Earth Science, University of Ferrara, via Saragat 1, I-44122, Ferrara, Italy

${ }^{5}$ Center for Interdisciplinary Exploration and Research in Astrophysics (CIERA) and Department of Physics and Astrophysics,

Northwestern University, Evanston, IL 60208, USA
${ }^{6}$ Department of Astronomy and Astrophysics, University of California, Santa Cruz, CA 95064, USA

${ }^{7}$ Steward Observatory, University of Arizona, 933 N. Cherry Avenue, Tucson, AZ 85721, USA

${ }^{8}$ The Observatories of the Carnegie Institution for Science, 813 Santa Barbara Street, Pasadena, CA 91101, USA ${ }^{9}$ Department of Physics, University of Bath, Claverton Down, Bath, BA2 7AY, UK

${ }^{10}$ Astrophysics Research Institute, Liverpool John Moores University, IC2, Liverpool Science Park, 146 Brownlow Hill, Liverpool L3 5RF, UK

${ }_{11}$ The Oskar Klein Centre \& Department of Astronomy, Stockholm University, AlbaNova, SE-106 91 Stockholm, Sweden

${ }_{12}^{12}$ Department of Astronomy, California Institute of Technology, 1200 East California Boulevard, Pasadena, CA 91125, USA

${ }^{13}$ Department of Physics and Astronomy, California State University, Sacramento, 6000 J Street, Sacramento, CA 95819, USA ${ }^{14}$ Max-Planck-Institut für Radioastronomie, Auf dem Huegel 69, D-53121 Bonn, Germany

${ }^{15}$ Center for Gravitational Physics, Yukawa Institute for Theoretical Physics, Kyoto University, Kyoto 606-8502, Japan Received 2017 October 17; revised 2018 May 8; accepted 2018 June 4; published 2018 July 26

\begin{abstract}
We present detailed multiwavelength observations of GRB 161219B at $z=0.1475$, spanning the radio to X-ray regimes, and the first Atacama Large Millimeter/submillimeter Array (ALMA) light curve of a $\gamma$-ray burst (GRB) afterglow. The centimeter- and millimeter-band observations before 8.5 days require emission in excess of that produced by the afterglow forward shock (FS). These data are consistent with radiation from a refreshed reverse shock (RS) produced by the injection of energy into the FS, signatures of which are also present in the X-ray and optical light curves. We infer a constant-density circumburst environment with an extremely low density, $n_{0} \approx 3 \times 10^{-4} \mathrm{~cm}^{-3}$, and show that this is a characteristic of all strong RS detections to date. The Karl G. Lansky Very Large Array (VLA) observations exhibit unexpected rapid variability on roughly minute timescales, indicative of strong interstellar scintillation. The X-ray, ALMA, and VLA observations together constrain the jet break time, $t_{\text {jet }} \approx 32$ days, yielding a wide jet opening angle of $\theta_{\text {jet }} \approx 13^{\circ}$, implying beaming-corrected $\gamma$-ray and kinetic energies of $E_{\gamma} \approx 4.9 \times 10^{48} \mathrm{erg}$ and $E_{\mathrm{K}} \approx 1.3 \times 10^{50} \mathrm{erg}$, respectively. Comparing the RS and FS emission, we show that the ejecta are only weakly magnetized, with relative magnetization, $R_{\mathrm{B}} \approx 1$, compared to the FS. These direct, multifrequency measurements of a refreshed RS spanning the optical to radio bands highlight the impact of radio and millimeter data in probing the production and nature of GRB jets.
\end{abstract}

Key words: gamma-ray burst: general - gamma-ray burst: individual (GRB 161219B)

Supporting material: figure set, machine-readable tables

\section{Introduction}

Long-duration $\gamma$-ray bursts (GRBs) have thus far been almost exclusively discovered through their prompt $\gamma$-ray emission, which unequivocally arises from relativistic outflows at high Lorentz factors, $\Gamma \gtrsim 10^{2}$ (Krolik \& Pier 1991; Fenimore et al. 1993; Baring \& Harding 1995, 1997; Woods \& Loeb 1995; Lithwick \& Sari 2001). These outflows are understood to be produced by a nascent, compact central engine, such as a magnetar or accreting black hole, formed in the collapsing core of a dying massive star (Piran 2005; Woosley \& Bloom 2006; Metzger et al. 2011). The internal shock model proposed to explain the $\gamma$-ray emission invokes collisions between shells with a wide distribution of Lorentz factors ejected by the engine (Rees \& Meszaros 1992;

\footnotetext{
16 Jansky Fellow.

17 Hubble Fellow.

18 Hubble and Carnegie-Dunlap Fellow.
}

Kobayashi et al. 1997; Kumar \& Piran 2000). Understanding the distribution of ejecta energy as a function of their Lorentz factor is therefore a critical probe of the nature of the central engine, its energy source, and the energy extraction mechanism (Woosley 1993; MacFadyen \& Woosley 1999; Aloy et al. 2000; Narayan et al. 2001; Zhang et al. 2003; Tchekhovskoy et al. 2008).

While monitoring the $\gamma$-ray sky remains an excellent means for detecting GRBs, a detailed description of the energetics of their jets and their progenitor environments is only possible through a study of the long-lasting X-ray to radio afterglow, generated when ejecta interact with their circumburst environment, setting up the forward shock (FS), and producing synchrotron radiation (Sari et al. 1998). Theoretical modeling of detailed multiwavelength observations in the synchrotron framework yields the energy of the explosion, the degree of jet collimation, the density of the surrounding medium, and the mass-loss history of the progenitor star, as 
well as information about the microphysical processes responsible for relativistic particle acceleration (Sari et al. 1999; Chevalier \& Li 2000; Granot \& Sari 2002).

Whereas GRB afterglows have traditionally been modeled as arising from jets with a uniform bulk Lorentz factor, radially structured ejecta profiles with energy spanning a range of Lorentz factors are gaining traction as viable models for the observed deviations of X-ray and optical light curves from the synchrotron model $^{19}$ (Björnsson et al. 2002, 2004; Nakar \& Piran 2003; Huang et al. 2006; Jóhannesson et al. 2006; Melandri et al. 2008, 2009; Troja et al. 2012; Virgili et al. 2013). Ejecta released later, or at lower Lorentz factors than the initial impulsive shell responsible for the prompt emission, catch up with the contact discontinuity during the afterglow phase and inject energy into the FS (Rees \& Meszaros 1998; Sari \& Mészáros 2000). Energy injection through massive ejecta may explain late-time plateaus, rebrightening events, slow decays, and unexpected breaks observed in the X-ray and optical light curves of some afterglows (Kumar \& Panaitescu 2000; Zhang \& Mészáros 2002; Granot et al. 2003; Panaitescu et al. 2006; Guidorzi et al. 2007; Mangano et al. 2007; Margutti et al. 2010a; Holland et al. 2012; Li et al. 2012; Greiner et al. 2013; Panaitescu et al. 2013; Nardini et al. 2014; de Pasquale et al. 2015; Beniamini \& Mochkovitch 2017) and forms a distinct class of models from late-time central engine activity, which has been invoked to explain some rapid X-ray and optical flares (Burrows et al. 2005b; Ioka et al. 2005; Ghisellini et al. 2009; Margutti et al. 2010b, 2011; Nardini et al. 2010; Li et al. 2012).

The process of energy transfer between the ejecta and the circumburst medium is expected to be mediated by a reverse shock (RS) propagating into the ejecta during the injection period. This RS is similar to the one expected from the deceleration of the ejecta by the circumburst environment as observed in exquisite detail in the afterglow of GRB 130427A (Laskar et al. 2013; Perley et al. 2014; van der Horst et al. 2014); however, an RS supported by energy injection is expected to continue propagating into the ejecta during the entire injection period (Zhang \& Mészáros 2002). If injection takes place in the form of a violent shell collision, the resulting strong RS is expected to exhibit a detectable observational signature in the form of an optical flash or radio flare (Akerlof et al. 1999; Kulkarni et al. 1999; Sari \& Piran 1999a; Soderberg \& Ramirez-Ruiz 2002; Zhang \& Mészáros 2002; Berger et al. 2003; Kobayashi \& Zhang 2003; Soderberg \& RamirezRuiz 2003; Chevalier et al. 2004). In the case of gentle or continuous energy injection, the RS is long-lasting, and its flux remains proportional to that of the FS during the entire injection period, $F_{\nu, \mathrm{m}, \mathrm{r}} \propto \Gamma F_{\nu, \mathrm{m}, \mathrm{f}}$ (Sari \& Mészáros 2000; Zhang \& Mészáros 2002; Panaitescu \& Kumar 2004; Genet et al. 2007; Lyutikov \& Camilo Jaramillo 2017). Thus, it may be possible to detect RSs arising from energy injection in cases both of violent collisions and of interactions at high enough ejecta Lorentz factor. Strong RS signatures are also excellent probes of the magnetization of the jets $\left(\sigma_{\mathrm{B}}\right)$, since high $\sigma_{\mathrm{B}}$ effectively increases the sound speed, ${ }^{20}$ thereby suppressing shock formation (Giannios et al. 2008).

\footnotetext{
19 Alternate explanations include circumburst density enhancements, structured jets, viewing angle effects, varying microphysical parameters, and gravitational microlensing (Eichler \& Granot 2006; Granot et al. 2006; Nousek et al. 2006; Panaitescu et al. 2006; Toma et al. 2006; Zhang et al. 2006; Jin et al. 2007; Shao \& Dai 2007; Kong et al. 2010; Uhm \& Zhang 2014; Duffell \& MacFadyen 2015).

${ }^{20}$ In magnetized media, information travels at the speed of the fast magnetosonic wave.
}

Our previous observations of GRB 140304A at $z \approx 5.3$ yielded the first multifrequency, multiepoch detection of an RS from a violent shell collision, lending credence to the multishell model (Laskar et al. 2018b). However, the high redshift of this event impacted the quality of data, limiting the strength of the inference feasible. In an analysis of four GRB afterglows exhibiting late-time optical and X-ray rebrightening events, we constrained the distribution of ejecta energy as a function of Lorentz factor (Laskar et al. 2015). In one case, our observations were incompatible with $\mathrm{RS}$ radiation from the injection, suggesting that collisions in at least some instances may be gentle processes; for the remaining three cases, the observations lacked the requisite temporal sampling and frequency coverage to conclusively rule out an injection RS. The reason may partly stem from the fact that the RS emission peaks in the millimeter band for typical shock parameters, and no facilities in this observing window had the requisite sensitivity (de Ugarte Postigo et al. 2012). However, the advent of the Atacama Large Millimeter/submillimeter Array (ALMA) now allows us to track the millimeter-band evolution of afterglows to a sensitivity of $\sim 30-100 \mu \mathrm{Jy}$ for the first time, reenergizing the search for refreshed RSs.

Here we report detailed radio through X-ray observations of GRB 161219B at $z=0.1475$ and present the first ALMA light curve of a GRB afterglow. The centimeter-band spectral energy distributions (SEDs) at $\lesssim 8.5$ days exhibit unusual spectral features, which we discuss in detail in a separate work (Alexander et al. 2018, hereafter ALB18). Through multiwavelength modeling of the X-ray, optical, and late radio data, we constrain the parameters of the FS powering the afterglow emission. The resulting model overpredicts the early X-ray emission, which can be explained by an episode of energy injection culminating at $\approx 0.25$ days. We interpret the early optical and radio observations as arising from an RS launched by the same injection event. By tying the RS and FS parameters together, we show that the ejecta were not strongly magnetized. We employ standard cosmological parameters of $\Omega_{m}=0.31$, $\Omega_{\lambda}=0.69$, and $H_{0}=68 \mathrm{~km} \mathrm{~s}^{-1} \mathrm{Mpc}^{-1}$. All magnitudes are in the $\mathrm{AB}$ system and not corrected for Galactic extinction, ${ }^{21}$ all uncertainties are at $1 \sigma$, and all times are relative to the Swift trigger time and in the observer frame, unless otherwise indicated.

\section{GRB Properties and Observations}

GRB 161219B was discovered by the Swift (Gehrels et al. 2004) Burst Alert Telescope (BAT, Barthelmy et al. 2005) on 2016 December 19 at 18:48:39 UT (D'Ai et al. 2016). The burst duration is $T_{90}=6.94 \pm 0.79 \mathrm{~s}$, and the $\gamma$-ray spectrum is well fit with a power-law plus exponential cutoff model $^{22}$

$$
\frac{d N_{\gamma}}{d E_{\gamma}}=E_{\gamma}^{\alpha_{\gamma}} e^{-E_{\gamma}\left(2+\alpha_{\gamma}\right) / E_{\gamma, \text { peak }},}
$$

with power-law photon index $\alpha_{\gamma}=-1.29 \pm 0.35$ and $E_{\gamma \text {,peak }}=$ $61.9 \pm 16.5 \mathrm{keV}$, yielding a fluence of $F_{\gamma}=(1.5 \pm 0.1) \times$ $10^{-6} \mathrm{erg} \mathrm{cm}^{-2} \quad(15-150 \mathrm{keV}, 90 \%$ confidence; Palmer et al. 2016). The burst was also detected by Konus-Wind with a

\footnotetext{
21 Galactic extinction correction based on Schlafly \& Finkbeiner (2011) is built into our modeling software (Laskar et al. 2014).

${ }^{22}$ Here $d N_{\gamma}$ is the number of photons with energy in the range $E$ to $E+d E$.
} 
duration of $T_{90} \approx 10 \mathrm{~s}$; the spectral fit to the Konus-Wind light curve yields $\alpha_{\gamma}=-1.59 \pm 0.71, E_{\text {peak }}=91 \pm 21 \mathrm{keV}$, and $F_{\gamma}=(3.1 \pm 0.8) \times 10^{-6} \mathrm{erg} \mathrm{cm}^{-2}(20-1000 \mathrm{keV}, 1 \sigma$; Frederiks et al. 2016). The optical afterglow was discovered by the Swift UV/Optical Telescope (UVOT; Roming et al. 2005) in observations beginning $112 \mathrm{~s}$ after the BAT trigger (Marshall \& D'Ai 2016). Spectroscopic observations $36 \mathrm{hr}$ after the burst with the X-shooter instrument on the European Southern Observatory (ESO) $8.2 \mathrm{~m}$ Very Large Telescope (VLT) provided a redshift of $z=0.1475$ (Tanvir et al. 2016). At this redshift, the inferred isotropic-equivalent $\gamma$-ray energy in the $1-10^{4} \mathrm{keV}$ rest-frame energy band is $E_{\gamma \text {,iso }}=(1.8 \pm 0.4) \times 10^{50} \mathrm{erg}$ from KonusWind and $E_{\gamma, \text { iso }}=(1.1 \pm 0.1) \times 10^{50} \mathrm{erg}$ from Swift-BAT, respectively, based on a Monte Carlo analysis using the respective spectral parameters. Since the Konus-Wind energy range is wider than the BAT band and therefore samples more of the $\gamma$-ray spectrum, we use the value of $E_{\gamma, \text { iso }}$ as determined from KonusWind in this work. The corresponding isotropic-equivalent luminosity is $L_{\gamma, \text { iso }}=E_{\gamma, \text { iso }}(1+z) T_{90}^{-1} \approx 10^{49} \mathrm{erg} \mathrm{s}^{-1}$, which makes this an intermediate-luminosity GRB (Bromberg et al. 2011).

\subsection{X-Ray: Swift/XRT}

The Swift X-ray Telescope (XRT; Burrows et al. 2005a) began observing GRB 161219B $108 \mathrm{~s}$ after the BAT trigger. The X-ray afterglow was localized to R.A. $=06^{\mathrm{h}} 06^{\mathrm{m}} 51^{\mathrm{s}} .37$, decl. $=-26^{\circ} 47^{\prime} 299^{\prime \prime} 7$ (J2000), with an uncertainty radius of 1 !. 4 (90\% containment). ${ }^{23}$ XRT continued observing the afterglow for 123 days in photon counting mode.

We extract XRT PC-mode spectra using the online tool on the Swift website (Evans et al. 2007, 2009). ${ }^{24}$ We downloaded the event and response files and fit them using the HEASOFT (v6.19) software package and corresponding calibration files. We used Xspec to fit the data, assuming a photoelectrically absorbed power-law model (tbabs $\times$ ztbabs $\times$ pow), constraining the intrinsic absorption to remain constant across the epochs, and fixing the Galactic absorption column to $N_{\mathrm{H}, \mathrm{Gal}}=3.06 \times 10^{20} \mathrm{~cm}^{-2}$ (Willingale et al. 2013). We do not find strong evidence for evolution in the $\mathrm{X}$-ray photon index. Constraining the photon index to remain fixed, we find $\Gamma_{X}=1.86 \pm 0.03$ for a spectrum comprising all available PC-mode data (Table 1). We use this value of the photon index and the unabsorbed counts-to-flux conversion rate from the Swift website of $4.95 \times 10^{-11} \mathrm{erg} \mathrm{cm}^{-2}$ count $^{-1}$ to convert the $0.3-10 \mathrm{keV}$ count-rate light curve $\mathrm{e}^{25}$ to flux density at $1 \mathrm{keV}$ for subsequent analysis. We combine the uncertainty in flux calibration based on our spectral analysis $(2.4 \%)$ in quadrature with the statistical uncertainty from the online light curve.

\subsection{UV, Optical, and Near-IR}

We analyzed the UVOT data using HEASOFT (v. 6.19) and corresponding calibration files. The afterglow was detected in all seven optical and UV filters. The background near the source was dominated by diffracted lighted from a nearby

\footnotetext{
23 http://www.swift.ac.uk/xrt_positions/727541/

24 http://www.swift.ac.uk/xrt_spectra/727541/

25 Obtained from the Swift website at http://www.swift.ac.uk/xrt_curves/ 727541 and rebinned to a minimum signal-to-noise ratio per bin of 8 .
}

Table 1

XRT Spectral Analysis for GRB 161219B

\begin{tabular}{lc}
\hline \hline Parameter & Value \\
\hline$T_{\text {start }}(\mathrm{s})$ & $1.1 \times 10^{2}$ \\
$T_{\text {end }}(\mathrm{s})$ & $1.1 \times 10^{7}$ \\
$N_{\mathrm{H}, \text { gal }}\left(10^{20} \mathrm{~cm}^{-2}\right)$ & 3.06 \\
$N_{\mathrm{H}, \text { int }}\left(10^{21} \mathrm{~cm}^{-2}\right)$ & $2.2 \pm 0.1$ \\
Photon index, $\Gamma_{\mathrm{X}}$ & $1.86 \pm 0.03$ \\
Flux $^{\mathrm{a}}$ (observed) & $(1.86 \pm 0.05) \times 10^{-12}$ \\
Flux $^{\mathrm{a}}$ (unabsorbed) & $(2.41 \pm 0.06) \times 10^{-12 \mathrm{~b}}$ \\
$C$-statistic (dof) & $684(699)$ \\
\hline
\end{tabular}

Notes.

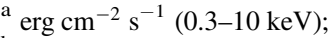

b Assuming the same fractional uncertainty as for the absorbed flux.

Table 2

Swift UVOT Observations of GRB 161219B

\begin{tabular}{lcccc}
\hline \hline $\begin{array}{l}\text { Mid-time, } \\
\Delta t \text { (days) }\end{array}$ & $\begin{array}{c}\text { UVOT } \\
\text { Band }\end{array}$ & $\begin{array}{c}\text { Flux Density } \\
(\mathrm{mJy})\end{array}$ & $\begin{array}{c}\text { Uncertainty } \\
(\mathrm{mJy})\end{array}$ & $\begin{array}{c}\text { Detection? } \\
(1=\text { Yes })\end{array}$ \\
\hline $2.16 \times 10^{-3}$ & $u w h$ & $6.19 \times 10^{-1}$ & $1.74 \times 10^{-2}$ & 1 \\
$5.19 \times 10^{-3}$ & $u v u$ & $5.97 \times 10^{-1}$ & $1.67 \times 10^{-2}$ & 1 \\
$6.81 \times 10^{-3}$ & $u v b$ & $6.92 \times 10^{-1}$ & $7.38 \times 10^{-2}$ & 1 \\
$7.10 \times 10^{-3}$ & $u w h$ & $5.30 \times 10^{-1}$ & $2.50 \times 10^{-2}$ & 1 \\
$7.39 \times 10^{-3}$ & $u w 2$ & $2.99 \times 10^{-1}$ & $4.12 \times 10^{-2}$ & 1 \\
\hline
\end{tabular}

(This table is available in its entirety in machine-readable form.)

$R \sim 13$ mag USNO-B1 star (R.A. $=06^{\mathrm{h}} 06^{\mathrm{m}} 50.65$, decl. $=$ $-26^{\circ} 47^{\prime} 53$ !" 3 ; J2000) $21^{\prime \prime}$ SE of the afterglow. We performed photometry using the recommended $5^{\prime \prime}$ aperture centered on the source but estimated the background contribution using an annulus with inner radius $21^{\prime \prime}$ and outer radius $31^{\prime \prime}$ centered on the nearby star, masking out one other contaminating source from the background region. The uncertainty in the background measurement contributes an additional, unknown source of systematic uncertainty in the target flux density near the end of the UVOT light curve (Table 2).

We began observing GRB 161219B with two $1 \mathrm{~m}$ telescopes in Sutherland (South Africa), which are operated by the Las Cumbres Observatory Global Network (LCOGT; Brown et al. 2013), on 2016 December 19, 20:43 UT, at $1.9 \mathrm{hr}$ since the GRB, in Sloan Digital Sky Survey (SDSS) $r^{\prime}$ and $i^{\prime}$ filters. Observations with 1 and $2 \mathrm{~m}$ LCOGT telescopes (formerly Faulkes Telescopes North and South) both in Hawaii and in Siding Springs (Australia) proceeded on a daily basis for 4 days, followed by a regularly increasing spacing until 2017 January 14 (25 days post-GRB). Additional optical observations with the $2 \mathrm{~m}$ Liverpool Telescope (LT; Steele et al. 2004) in the same filters culminated on January 23 (35 days postGRB). Bias and flat-field corrections were applied using the specific pipelines of the LCOGT and of the LT. The optical afterglow magnitudes were obtained by PSF-fitting photometry, after calibrating the zero-points with four nearby stars with SDSS $r^{\prime}$ and $i^{\prime}$ magnitudes from the AAVSO Photometric All-Sky Survey (APASS) catalog (Henden et al. 2016). A systematic error of 0.02 mag due to the zero-point scatter of the calibrating stars was incorporated as an additional source of uncertainty in the magnitudes. 
Table 3

Optical and Near-IR Observations of GRB 161219B

\begin{tabular}{|c|c|c|c|c|c|c|c|c|}
\hline $\begin{array}{l}\Delta t \\
\text { (days) }\end{array}$ & Observatory & Instrument & Filter & $\begin{array}{c}\text { Frequency } \\
(\mathrm{Hz})\end{array}$ & $\begin{array}{l}\text { Flux Density } \\
(\mathrm{mJy})\end{array}$ & $\begin{array}{c}\text { Uncertainty }^{\mathrm{a}} \\
(\mathrm{mJy})\end{array}$ & $\begin{array}{c}\text { Detection? } \\
1=\text { Yes }\end{array}$ & Reference \\
\hline $5.44 \times 10^{-4}$ & SAAO & MASTER & $C R$ & $4.67 \times 10^{14}$ & $1.34 \times 10^{0}$ & $4.26 \times 10^{-1}$ & 1 & Buckley et al. (2016) \\
\hline $7.33 \times 10^{-3}$ & Terksol & K-800 & $C R$ & $4.67 \times 10^{14}$ & $6.52 \times 10^{-1}$ & $2.35 \times 10^{-2}$ & 1 & Mazaeva et al. (2016) \\
\hline $8.82 \times 10^{-3}$ & Terksol & K-800 & $C R$ & $4.67 \times 10^{14}$ & $7.29 \times 10^{-1}$ & $2.81 \times 10^{-2}$ & 1 & Mazaeva et al. (2016) \\
\hline$\ldots$ & $\ldots$ & $\ldots$ & $\ldots$ & $\ldots$ & $\ldots$ & $\ldots$ & .. & $\ldots$ \\
\hline
\end{tabular}

Note.

a An uncertainty of $0.2 \mathrm{AB}$ mag is assumed where not provided. The data have not been corrected for Galactic extinction.

(This table is available in its entirety in machine-readable form.)

We obtained uBVgri imaging of GRB 161219B from 2016 December 22 to 2017 March 21 using the Direct CCD Camera on the Swope $1.0 \mathrm{~m}$ telescope at Las Campanas Observatory in Chile. We reduced the data using the photpipe imaging and photometry package (Rest et al. 2005) following the methods described in Kilpatrick et al. (2018). We performed aperture photometry using a $4^{\prime \prime}$ circular aperture on the position of GRB 161219B. We calibrated the photometry in $u^{\prime}$ band using Tycho2 standards and the other filters using PS1 standard-star fields observed in the same instrumental configuration and at a similar airmass, after transforming the gri magnitudes to the Swope natural system using the corresponding filter functions (Scolnic et al. 2015).

We observed GRB 161219B with the Low Resolution Imaging Spectrometer (Oke et al. 1995) on the $10 \mathrm{~m}$ Keck I telescope on 2017 March 29 in UBgRIz bands. The images were bias-subtracted, flat-fielded, and cleaned of cosmic rays using LPipe. ${ }^{26}$ The host galaxy is well detected in all filters. We performed photometry relative to the PS1, Tycho2, and APASS standards using a $4^{\prime \prime}$ aperture.

We obtained seven epochs of near-IR observations in the $J H K$ bands with the Wide-field Camera (WFCAM; Casali et al. 2007) mounted on the United Kingdom Infrared Telescope (UKIRT) spanning $\approx 2.5$ to $\approx 270$ days. We obtained pre-processed images from the WFCAM Science Archive (Hamly et al. 2008) that are corrected for bias, flat-field, and dark current by the Cambridge Astronomical Survey Unit. ${ }^{27}$ For each epoch and filter, we co-add the images and perform astrometry relative to the Two Micron All Sky Survey using a combination of tasks in Starlink ${ }^{28}$ and IRAF. We perform aperture photometry using standard tasks in IRAF using an aperture of 4.5 times the FWHM of the seeing measured from stars in the field, in order to capture the combined light of the afterglow, supernova (SN), and host galaxy.

We present the results of our optical and near-IR (NIR) photometry, together with a compilation of all other optical observations reported in GCN circulars in Table 3. We include the GROND, Gran Telescopio Canarias (GTC), Nordic Optical Telescope (NOT), and Pan-STARRS1 (PS1) light curves presented by Cano et al. (2017a), together with the ESO VLT, the $3.6 \mathrm{~m}$ Telescopio Nazionale Galileo, LT, and Keck observations presented by Ashall et al. (2017) in our modeling.

\footnotetext{
$\overline{26}$ http://www.astro.caltech.edu/dperley/programs/lpipe.html

27 http://casu.ast.cam.ac.uk/

${ }^{28}$ http://starlink.eao.hawaii.edu/starlink
}

\subsection{Millimeter: ALMA}

We obtained ALMA observations of the afterglow at 1.3 days after the burst through program 2016.1.00819.T (PI: Laskar) in Band 3, with two $4 \mathrm{GHz}$-wide basebands centered at 91.5 and $103.5 \mathrm{GHz}$, respectively. Prompt data reduction, facilitated through rapid data release by the Joint ALMA Observatory (JAO), yielded a strong $(\gtrsim 50 \sigma)$ detection in our 31-minute scheduling block, with 16 minutes on source. We acquired two additional epochs with an identical setup at $\approx 3.3$ and $\approx 8.3$ days, respectively. Given the brightness of the afterglow and the unusual nature of the radio SEDs, we requested and were granted Director's Discretionary Time through program 2016.A.00015.S for two additional epochs. All observations utilized J0522-3627 as the bandpass and flux density calibrator and J0614-2536 as the phase calibrator. We imaged the pipeline products using the Common Astronomy Software Application (CASA; McMullin et al. 2007). The afterglow was detected in all five epochs, and the superb sensitivity of ALMA allowed us to measure the flux density in the two sidebands separately, yielding the first ALMA light curve of a GRB afterglow. We report the results of our ALMA observations in Table 4.

\subsection{Centimeter: VLA}

We observed the afterglow using the Karl G. Jansky Very Large Array (VLA) starting 0.5 days after the burst through program 15A-235 (PI: Berger). We detected and tracked the flux density of the afterglow from 1.2 to $37 \mathrm{GHz}$ over nine epochs until $\approx 159$ days after the burst. We used $3 \mathrm{C} 48$ as the flux and bandpass calibrator and J0608-2220 as the gain calibrator. Some of the high-frequency observations $(\gtrsim 15 \mathrm{GHz})$ suffered from residual phase errors, which we remedied using phase-only self-calibration in epochs with sufficient signal-tonoise ratio. We carried out data reduction using CASA and list the results of our VLA observations in Table 5.

\section{Basic Considerations}

We now interpret the X-ray, optical, and radio observations in the standard synchrotron framework (Sari et al. 1998; Granot \& Sari 2002), in which the observed spectra are characterized by power-law segments connected at characteristic break frequencies: the self-absorption frequency $\left(\nu_{\mathrm{a}}\right)$, the characteristic synchrotron frequency $\left(\nu_{\mathrm{m}}\right)$, and the cooling frequency $\left(\nu_{\mathrm{c}}\right)$. The electrons responsible for the observed radiation are assumed to form a power-law distribution in energy with index $p$. 
Table 4

GRB 161219B: Log of ALMA Observations

\begin{tabular}{lccc}
\hline \hline $\begin{array}{l}\Delta t \\
\text { (days) }\end{array}$ & $\begin{array}{c}\text { Frequency } \\
(\mathrm{GHz})\end{array}$ & $\begin{array}{c}\text { Flux Density } \\
(\mu \mathrm{Jy})\end{array}$ & $\begin{array}{c}\text { Uncertainty } \\
(\mu \mathrm{Jy})\end{array}$ \\
\hline 1.30 & 91.5 & 1332 & 32 \\
1.30 & 103.5 & 1244 & 31 \\
3.30 & 91.5 & 853 & 34 \\
3.30 & 103.5 & 897 & 33 \\
8.31 & 91.5 & 505 & 15 \\
8.31 & 103.5 & 500 & 19 \\
24.45 & 91.5 & 314 & 41 \\
24.45 & 103.5 & 285 & 43 \\
78.18 & 91.5 & 64 & 14 \\
78.18 & 103.5 & 51 & 20 \\
\hline
\end{tabular}

Table 5

GRB 161219B: Log of VLA Observations

\begin{tabular}{lcccc}
\hline \hline $\begin{array}{l}\Delta t \\
(\text { days })\end{array}$ & $\begin{array}{c}\text { Frequency } \\
(\mathrm{GHz})\end{array}$ & $\begin{array}{c}\text { Flux Density } \\
(\mu \mathrm{Jy})\end{array}$ & $\begin{array}{c}\text { Uncertainty } \\
(\mu \mathrm{Jy})\end{array}$ & Det.? \\
\hline 0.51 & 19.0 & 278.1 & 28.6 & 1 \\
0.51 & 21.0 & 156.2 & 41.7 & 1 \\
0.51 & 23.0 & 184.7 & 36.1 & 1 \\
0.51 & 25.0 & 242.4 & 47.5 & 1 \\
$\cdots$ & $\cdots$ & $\cdots$ & $\cdots$ & $\cdots$ \\
\hline
\end{tabular}

Note. The last column indicates a detection (1) or nondetection ( 0 ); in the latter case, the flux density is a $3 \sigma$ upper limit, and the uncertainty refers to the mean map noise. We include the Giant Metrewave Radio Telescope detection reported by Nayana \& Chandra (2016).

(This table is available in its entirety in machine-readable form.)

\subsection{X-Rays and Optical—and Location of $\nu_{\mathrm{c}}$}

The XRT light curve exhibits a flare at $4.2 \times 10^{-3}$ to $6.2 \times 10^{-3}$ days (Figure 1). Such flares in early X-ray light curves are relatively common and may arise from residual central engine activity or the collisions of relativistic shells (Burrows et al. 2007; Margutti et al. 2010b; Laskar et al. 2018b), and we do not include this portion of the light curve in our multiwavelength analysis. The PC-mode light curve after $8.8 \times 10^{-3}$ days can be fit with a power law with two breaks, described by

$$
\begin{aligned}
F_{\nu}(t)= & F_{\mathrm{b}}\left[\frac{1}{2}\left(\frac{t}{t_{\mathrm{b}, 1}}\right)^{-y_{1} \alpha_{\mathrm{X}, 1}}+\frac{1}{2}\left(\frac{t}{t_{\mathrm{b}, 1}}\right)^{-y_{1} \alpha_{\mathrm{X}, 2}}\right]^{-1 / y_{1}} \\
& \times\left[1+\left(\frac{t}{t_{\mathrm{b}, 2}}\right)^{y_{2}\left(\alpha_{\mathrm{X}, 2}-\alpha_{\mathrm{X}, 3}\right)}\right]^{-1 / y_{2}}
\end{aligned}
$$

breaking $^{29}$ first from $\alpha_{\mathrm{X}, 1}=-0.37 \pm 0.09$ to $\alpha_{\mathrm{X}, 2}=-0.82 \pm$ 0.02 at $\approx 0.06$ days and then to $\alpha_{\mathrm{X}, 3}=-1.32 \pm 0.08$ at $\approx 13$ days (Table 6 ). We also fit the Swift/UVOT light curves in three well-sampled bands at $\lesssim 2.4$ days ( $u w h, u v w 1, u v w 2)$ with a broken power-law model,

$$
F_{\nu}(t)=F_{\mathrm{b}}\left[\frac{1}{2}\left(\frac{t}{t_{\mathrm{b}}}\right)^{-y \alpha_{1}}+\frac{1}{2}\left(\frac{t}{t_{\mathrm{b}}}\right)^{-y \alpha_{2}}\right]^{-1 / y},
$$

\footnotetext{
${ }^{29}$ We fix the smoothness parameters here and in Equation (3) at $y_{1}=y_{2}=y=5$, and we use the convention $F_{\nu} \propto t^{\alpha} \nu^{\beta}$.
}

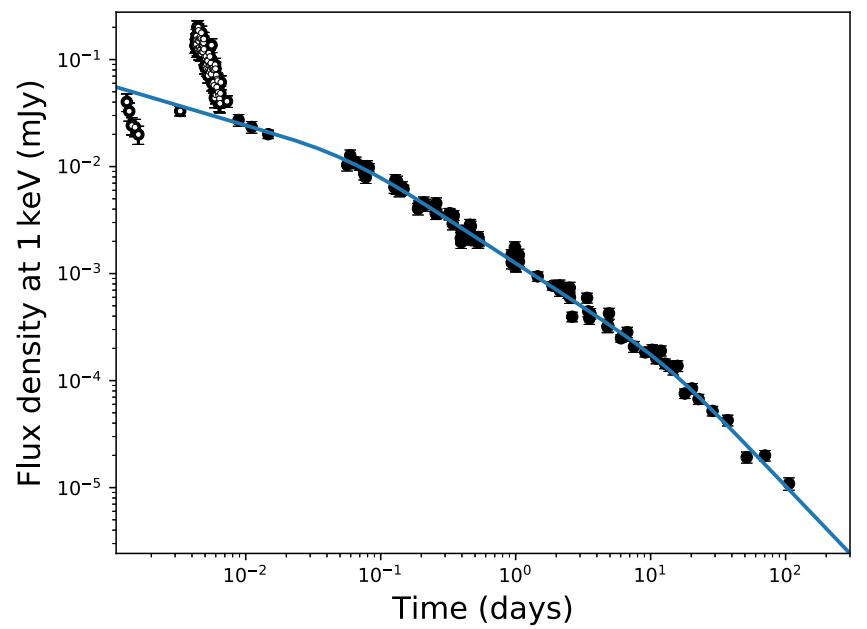

Figure 1. Swift XRT light curve of GRB 161219B at $1 \mathrm{keV}$ (black symbols), together with a twice-broken power-law fit (blue; Equation (2)). Data before $\approx 8.8 \times 10^{-3}$ days are dominated by flaring activity and are not included in the fit.

Table 6

XRT Light-curve Fit

\begin{tabular}{lc}
\hline \hline Parameter & Value \\
\hline$t_{\mathrm{b}, 1}$ (days) & $(6.0 \pm 2.3) \times 10^{-2}$ \\
$t_{\mathrm{b}, 2}$ (days) & $(1.3 \pm 0.4) \times 10^{1}$ \\
$F_{\mathrm{b}}(\mathrm{mJy})$ & $(9.7 \pm 2.6) \times 10^{-3}$ \\
$\alpha_{\mathrm{X}, 1}$ & $-0.37 \pm 0.09$ \\
$\alpha_{\mathrm{X}, 2}$ & $-0.82 \pm 0.02$ \\
$\alpha_{\mathrm{X}, 3}$ & $-1.32 \pm 0.08$ \\
$\chi^{2} /$ dof & $126 / 75$ \\
\hline
\end{tabular}

and provide a fit to the $r^{\prime}$ in this same period for reference in Table 7. The Swift/UVOT light curves in these three bands exhibit a shallow decline with $\alpha_{\mathrm{UV}, \text { avg, } 1}=-0.22 \pm 0.02$, followed by a steepening with $\alpha_{\mathrm{UV}, \text { avg, } 2}=-0.76 \pm 0.02$ at $t_{\mathrm{b}, \mathrm{UV} \text {,avg }}=(9.4 \pm$ 1.3) $\times 10^{-2}$ days (weighted averages; Figure 2). The prominent rebrightening in the optical light curves after $\approx 2.4$ days is associated with an emerging supernova (SN 2016jca ${ }^{30}$ ), and the subsequent flattening at $\gtrsim 50$ days can be attributed to contamination from an underlying host galaxy, the latter also detectable as an extension in the optical images (Cano et al. 2017a).

The break time of $t_{\mathrm{b} \text {,avg }} \approx 9 \times 10^{-2}$ days in the UV light curves is consistent with the time of the first break in the $\mathrm{X}$-ray light curve at $t_{\mathrm{b}, \mathrm{X}, 1} \approx 6 \times 10^{-2}$ days. Such an achromatic break is unusual in GRB afterglows, and in the standard synchrotron model it can only be explained as (i) onset of the afterglow (Sari \& Piran 1999b; Kobayashi \& Zhang 2007), (ii) viewing angle effects (Granot et al. 2002), and (iii) jet breaks (Rhoads 1999; Sari et al. 1999). Of these, the first two are preceded by rising light curves, and the third results in a steeply decaying light curve $(\alpha \approx-p)$, neither of which is the case here. We investigate the origin of this feature in Section 4.

We now interpret the observed light curves at $\gtrsim 0.1$ days in the synchrotron framework, beginning with the location of the cooling frequency, $\nu_{\mathrm{c}}$. We investigate four possibilities,

\footnotetext{
$\overline{30}$ https://wis-tns.weizmann.ac.il/object/2016jca/
} 
Table 7

UV/Optical Light-curve Fit

\begin{tabular}{lcccr}
\hline \hline Band & $\begin{array}{c}t_{\mathrm{b}} \\
(\mathrm{days})\end{array}$ & $\begin{array}{c}F_{\mathrm{b}} \\
(\mathrm{mJy})\end{array}$ & $\alpha_{1}$ & $\alpha_{2}$ \\
\hline$r^{\prime}$ & $0.15^{\mathrm{a}}$ & $0.31^{\mathrm{b}}$ & $-0.29 \pm 0.03$ & $-0.68 \pm 0.01$ \\
$u v w 1$ & $0.21 \pm 0.04$ & $0.13 \pm 0.02$ & $-0.33 \pm 0.04$ & $-0.95 \pm 0.05$ \\
$u v w 2$ & $0.12 \pm 0.03$ & $0.14 \pm 0.02$ & $-0.20 \pm 0.05$ & $-0.79 \pm 0.05$ \\
white & $0.07 \pm 0.02$ & $0.29 \pm 0.03$ & $-0.17 \pm 0.02$ & $-0.70 \pm 0.03$ \\
Avg $^{\mathrm{c}}$ & $0.094 \pm 0.013$ & $\ldots$ & $-0.22 \pm 0.02$ & $-0.76 \pm 0.02$ \\
\hline
\end{tabular}

Notes.

${ }^{a}$ Fixed.

b This parameter is strongly correlated with $t_{\mathrm{b}}$.

${ }^{c}$ Weighted average of the UV fits.

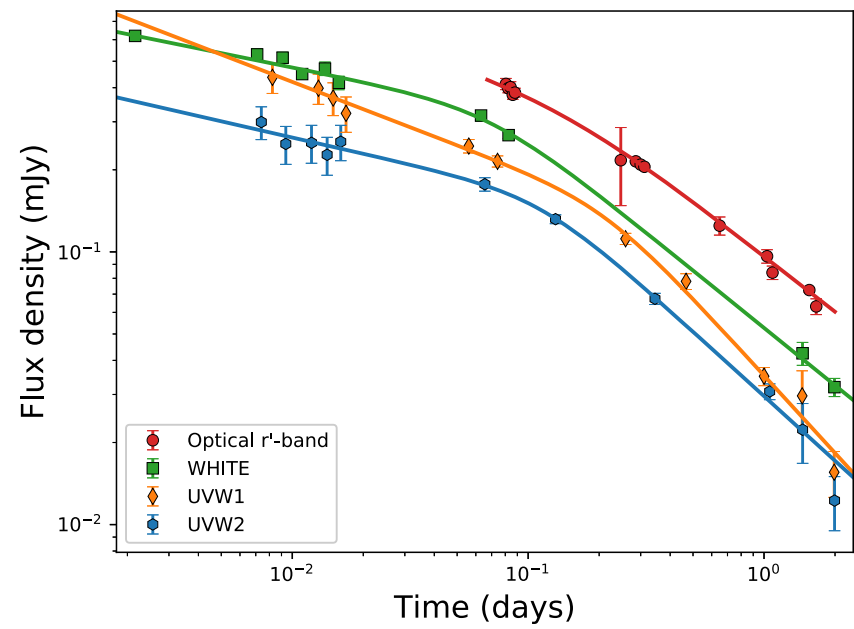

Figure 2. Optical $r^{\prime}$-band (red circles) and Swift/UVOT $u w h$-, $u w 1$-, and $u w 2$ band light curves of GRB 161219B (green squares, orange diamonds, and blue hexagons, respectively) before 2.4 days, together with broken power-law fits (lines; Equation (3)).

$\nu_{\mathrm{c}}>\nu_{\mathrm{X}}, \nu_{\mathrm{c}}<\nu_{\mathrm{opt}}, \nu_{\mathrm{opt}}<\nu_{\mathrm{c}}<\nu_{\mathrm{X}}$, and $\nu_{\mathrm{c}} \approx \nu_{\mathrm{X}}$. In the first scenario $\left(\nu_{\mathrm{c}}>\nu_{\mathrm{X}}\right)$, we note that the observed X-ray spectral index $\beta_{\mathrm{X}}=-0.86 \pm 0.03=(1-p) / 2$ implies $p=2.72 \pm$ 0.06 , which yields $\alpha_{\mathrm{X}}=-1.29 \pm 0.04$ (interstellar medium [ISM]) or $\alpha_{\mathrm{X}}=-1.79 \pm 0.04$ (wind). However, the measured decline rate is $\alpha_{\mathrm{X}}=-0.82 \pm 0.02$. Thus, $\nu_{\mathrm{c}}>\nu_{\mathrm{X}}$ between 0.1 days and $\approx 13$ days is ruled out.

If $\nu_{\mathrm{c}}<\nu_{\mathrm{opt}}$, then $\beta_{\mathrm{X}}=-0.86 \pm 0.03$ implies $p=1.76 \pm$ 0.06 . In this case, we expect the X-ray and optical to lie on the same spectral slope, with $\beta_{\text {opt }} \approx-0.86$. We find that the hostsubtracted GROND grizJHK photometry at $\approx 1.5$ days can be fit with a single power law, $\beta_{\text {opt }}=-0.5 \pm 0.1$. This is shallower than expected and cannot be explained if $\nu_{\mathrm{c}}<\nu_{\mathrm{opt}}$ (extinction in the host galaxy would further steepen the optical spectral index). Thus, $\nu_{\mathrm{c}}<\nu_{\mathrm{opt}}$ is ruled out.

If $\nu_{\mathrm{opt}}<\nu_{\mathrm{c}}<\nu_{\mathrm{X}}$, then the observed X-ray spectral index once again implies $p=1.76 \pm 0.06$. The expected optical spectral index is $(1-p) / 2=-0.38 \pm 0.03$, and the steeper slope could be explained as arising from extinction. For $p<2$, the expected light curves depend on assumptions regarding the normalization of the total energy of accelerated electrons relative to the energy of the FS (Bhattacharya 2001; Dai \& Cheng 2001). If we assume that the electron spectrum cuts off above a maximal electron Lorentz factor, ${ }^{31}$ that a constant fraction of the shock energy is given to the electrons, and that the total electron energy must be finite (Gao et al. 2013), then we would have $\alpha_{\mathrm{X}}=-(3 p+10) / 16=-0.96 \pm 0.01$, inconsistent with the observed value of $\alpha_{\mathrm{X}}=-0.82 \pm 0.02$, as well as $\alpha_{\mathrm{opt}}=-(3 p+2) / 16=-0.46 \pm 0.01$, inconsistent with the observed value of $\alpha_{\mathrm{opt}}=-0.76 \pm 0.02$. On the other hand, if we assume that the closure relations of Granot \& Sari (2002) apply for $p<2$, then we expect $\alpha_{\mathrm{X}}=(2-3 p) / 4=$ $-0.82 \pm 0.04$ and $\alpha_{\mathrm{opt}}=3(1-p) / 4=-0.57 \pm 0.04$ (ISM) or $\alpha_{\text {opt }}=(1-3 p) / 4=-1.07 \pm 0.04$ (wind). Whereas the observed X-ray decline rate matches this prediction, the optical decline rate does not. Thus, the $p=1.76 \pm 0.06$ model is disfavored.

We therefore investigate the last possibility, $\nu_{\mathrm{c}} \approx \nu_{\mathrm{X}}$ at $\gtrsim 0.1$ days. Anchoring this model to the optical spectral index, $\beta_{\mathrm{opt}}=-0.5 \pm 0.1$, we infer $p=1-2 \beta=2.0 \pm 0.2$ for the spectral ordering, $\nu_{\mathrm{m}}<\nu_{\mathrm{opt}}<\nu_{\mathrm{c}} \approx \nu_{\mathrm{X}}$. The observed UV spectral index, $\beta_{\mathrm{UV}}=-1.2 \pm 0.2$, is steeper than the optical and indicates extinction in the host galaxy. The observed optical decline rate of $\alpha_{\mathrm{opt}}=-0.76 \pm 0.02$ is not consistent with the predicted value of $\alpha_{\mathrm{opt}}=(1-3 p) / 4=-1.25 \pm$ 0.15 for the wind case, but it agrees with the expected value of $\alpha_{\mathrm{opt}}=3(1-p) / 4=-0.75 \pm 0.15$ for the ISM case.

The spectral index between the NIR $K$ band and the X-rays is $\beta_{\mathrm{ox}}=-0.68 \pm 0.02$; this is steeper than $\beta_{\mathrm{opt}}$ and consistent with $\nu_{\mathrm{opt}}<\nu_{\mathrm{c}}$ at this time. If $\nu_{\mathrm{c}} \approx \nu_{\mathrm{X}}$, we expect the X-ray spectral index to be intermediate between $(1-p) / 2 \approx-0.5$ and $-p / 2 \approx-1$, which is satisfied by the measured index, $\beta_{\mathrm{X}}=-0.86 \pm 0.03$. If we place $\nu_{\mathrm{c}} \approx 1 \mathrm{keV}$ and use the rounded shape of the cooling break as derived by Granot \& Sari (2002), we heuristically calculate a spectral index across the ends of the Swift X-ray band between 0.3 and $10 \mathrm{keV}$ of $\beta \approx-0.82$, consistent with the observed index. Finally, the observed decline rate, $\alpha_{\mathrm{X}}=-0.82 \pm 0.02$, is also intermediate between $3(1-p) / 4 \approx-0.75$ and $(2-3 p) / 4 \approx-1$, further indicating $\nu_{\mathrm{c}} \approx \nu_{\mathrm{X}}$.

Thus, the optical and $\mathrm{X}$-ray observations indicate an ISM environment with $\nu_{\mathrm{m}}<\nu_{\mathrm{opt}}<\nu_{\mathrm{c}} \approx \nu_{\mathrm{X}}$ at $\gtrsim 0.1$ days and moderate extinction in the host galaxy. Whereas the final

\footnotetext{
$\overline{31}$ One possibility for a high-energy cutoff in the electron spectrum $\left(\gamma_{\mathrm{M}}\right)$ is afforded by balancing the electron acceleration timescale and the dynamical timescale, $\gamma_{\mathrm{M}} \sim \Gamma t_{\mathrm{e}} B / m_{\mathrm{p}} c$, where $\Gamma$ is the shock Lorentz factor, $q_{\mathrm{e}}$ is the fundamental electron charge, $B$ is the post-shock magnetic field, $m_{\mathrm{p}}$ is the proton mass, and $c$ is the speed of light.
} 

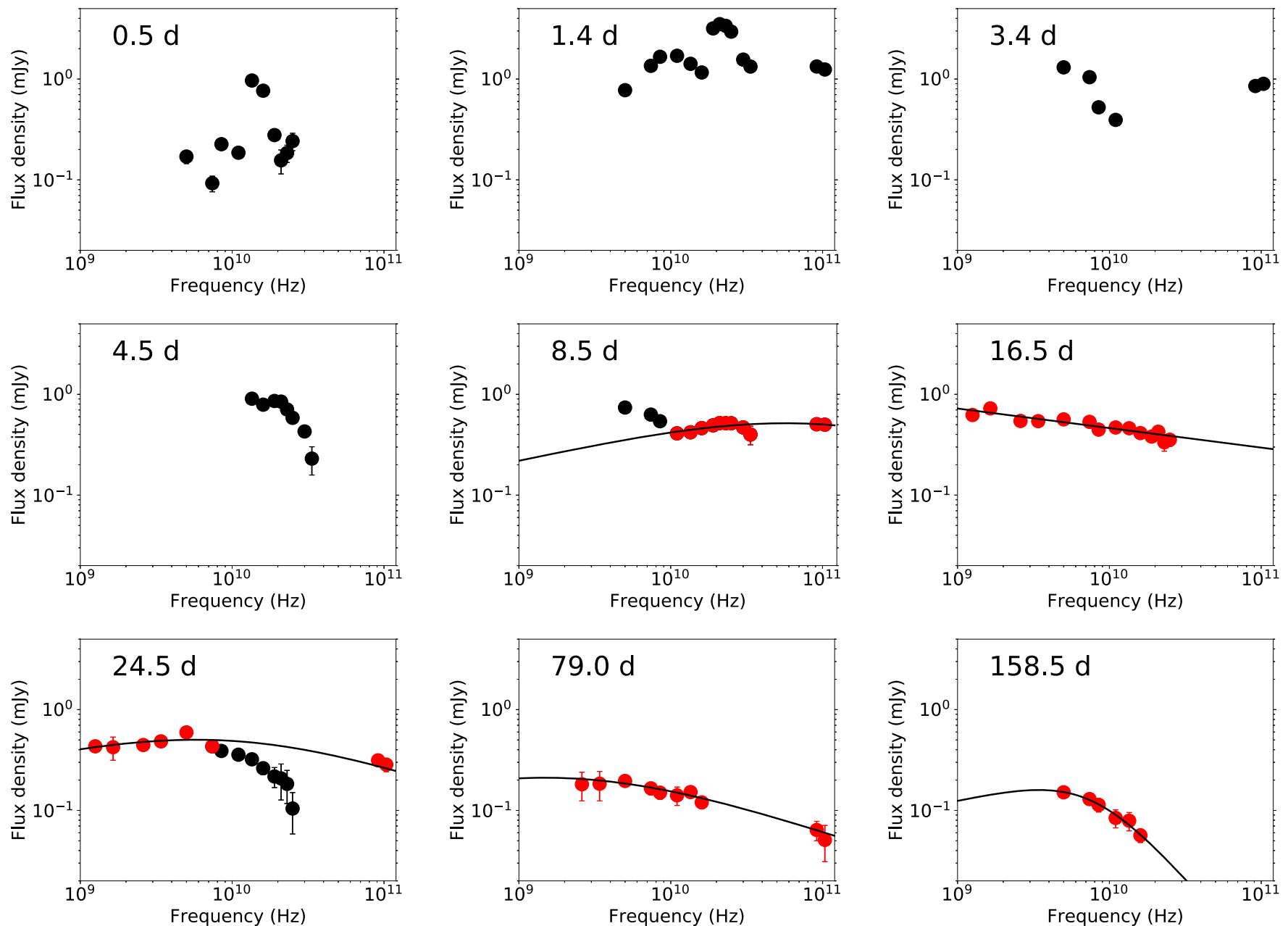

Figure 3. Multifrequency centimeter-band (VLA) and millimeter-band (ALMA) SEDs of the afterglow of GRB $161219 \mathrm{~B}$ from 0.5 to $\approx 159$ days, together with power-law (16.5 days) and broken power-law (8.5, 24.5, 79, and 158.5 days) fits (solid) to some of the observations (red circles; see Section 3.2 for details). The radio SEDs exhibit unexpected variability in the centimeter band (see also Figure 4).

X-ray decline rate of $\alpha_{\mathrm{X}, 3} \approx-1.3$ appears too shallow for a jet break, where we expect $\alpha_{\mathrm{X}} \approx-p \approx-2$ (Rhoads 1999; Sari et al. 1999), we note that the break time and post-break decay rate are degenerate with the break smoothness. A later break time in a physically motivated model may be consistent with the steeper post-break decay expected. Our subsequent multiwavelength analysis, described in Section 4, confirms this interpretation.

\subsection{Radio-Unexpected Variability and Location of $\nu_{\mathrm{m}}$}

The centimeter-band data of GRB 161219B are truly remarkable, exhibiting spectro-temporal variability on timescales and frequency scales shorter than ever observed for a GRB radio afterglow. The SEDs at 0.5, 1.4, 3.4, 4.5, and 8.5 days exhibit spectral features with $\delta \nu / \nu \lesssim 1$, too narrow for production via standard synchrotron emission (Figure 3 ). The observations at 24.5 days appear to exhibit a deficit at $\approx 5-30 \mathrm{GHz}$. Only the epochs at $\approx 16.5, \approx 79$, and $\approx 159$ days exhibit simple SEDs that can be understood as power laws, or combinations thereof.

These unexpected spectral features appear to be due to a combination of factors. At frequencies above $\approx 10 \mathrm{GHz}$, atmospheric phase decoherence can reduce the measured flux density. Whereas most of the data were obtained in
A-configuration and a fast cycle time of 2-4 minutes was employed, the phase referencing is not perfect and there are residual errors on all baselines. To check this, we performed phase-only self-calibration on the afterglow itself in epochs where the target was detected at a signal-to-noise ratio of $\gtrsim 3$ per solution interval and found the process to yield an increased flux density by $10 \%-30 \%$ and a reduced map noise in the vicinity of the afterglow. However, self-calibration is not possible when the target is fainter than $\approx 0.5-1 \mathrm{mJy}$, and even where feasible, this process does not completely explain the observed spectral features. We also find the observed rapid variability at centimeter bands to be robust to self-calibration.

High-cadence $u v$-domain fitting of the visibilities at time resolution of minutes reveals another unexpected variability: the afterglow light curve exhibits rapid brightening and fading within a single receiver baseband $(2 \mathrm{GHz}$ at $K$ band, $1 \mathrm{GHz}$ otherwise) in the first four epochs on timescales of minutes, while the spectral index across basebands within the same receiver tuning changes rapidly (Figure 4 ). The millimeter data do not exhibit comparable levels of variability, with the scatter in the time series being consistent with the mean uncertainty of the measurements. Whereas variability on short timescales is a known characteristic of diffractive interstellar scintillation, such effects have not been observed at frequencies $\gtrsim 10 \mathrm{GHz}$, as apparent for this event (Rickett 1990). A detailed discussion 

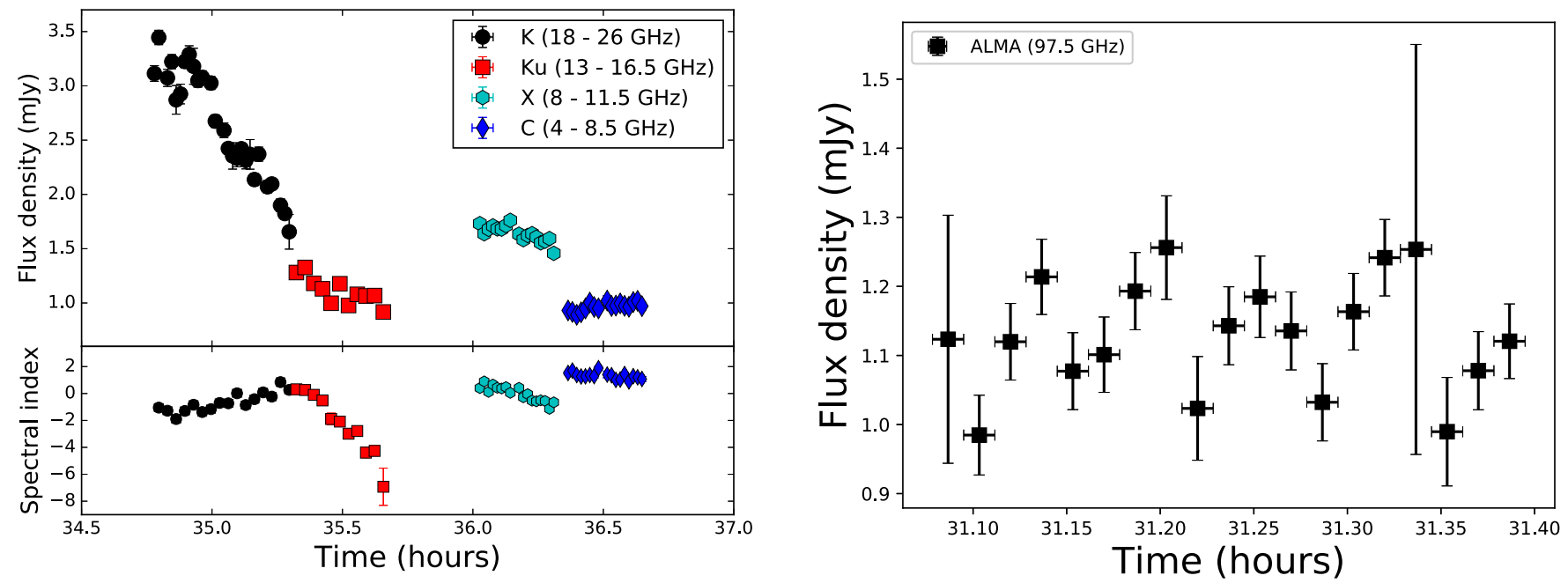

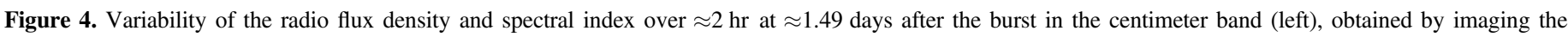

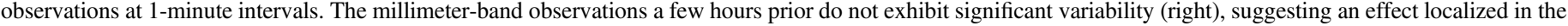
frequency domain.

of the centimeter-band variability is presented in ALB18; for the purposes of our broadband analysis, we use the timeaveraged data for each epoch, together with the ALMA light curve, to study the behavior of the afterglow in the centimeter and millimeter bands. The centimeter-band data in the first three epochs exhibit the greatest degree of variability, and we do not include them while computing the goodness of fit; however, they are important components for our final model, and we return to discussing the full centimeter-band data set in Section 6.

As the observed variability appears to decrease at $\gtrsim 8.5$ days, we attempt to derive the properties of the intrinsic emission by fitting the radio SEDs after this time. As the precise fits depend on the data selected for fitting, the true uncertainties on the measured numbers below are likely larger than those quoted, which are purely statistical.

The radio $\mathrm{SED}$ at $\approx 8.5$ days exhibits a rising spectrum at $\gtrsim 10 \mathrm{GHz}$. Fitting the data above $10 \mathrm{GHz}$ with a broken power law,

$$
F_{\nu}(\nu)=F_{\mathrm{b}}\left[\frac{1}{2}\left(\frac{\nu}{\nu_{\mathrm{b}}}\right)^{-y \beta_{1}}+\frac{1}{2}\left(\frac{\nu}{\nu_{\mathrm{b}}}\right)^{-y \beta_{2}}\right]^{-1 / y},
$$

fixing $\beta_{1}=1 / 3, \beta_{2}=(1-p) / 2 \approx-0.5$, and $y=1.84-$ $0.40 p \approx 1.0$ (appropriate for $\nu_{\mathrm{m}}$; Granot \& Sari 2002), yields $\nu_{\mathrm{b}}=(9.2 \pm 1.0) \times 10^{10} \mathrm{~Hz}$ with flux density $F_{\nu, \mathrm{pk}}=$ $0.508 \pm 0.007 \mathrm{mJy}$. The data at $\lesssim 10 \mathrm{GHz}$ are in excess of the $\nu^{1 / 3}$ power law, while the spectrum at 16.5 days is relatively flat and can be fit as a single power law with $\beta=-0.19 \pm 0.03$. We address both points together in Section 6 .

The SED at $\approx 24.5$ days exhibits a steep spectrum, $\beta=-1.1 \pm 0.2$ at $\approx 10-30 \mathrm{GHz}$, which underpredicts the ALMA observations at this time by a factor of $\approx 10$. It is possible that the decrement in the VLA observations at $\nu \gtrsim 10 \mathrm{GHz}$ relative to lower frequencies is due to phase decoherence, which systematically reduces the observed flux, ${ }^{32}$

\footnotetext{
32 If the phase fluctuations induced by the atmosphere on a given baseline can be approximated as a Gaussian random process with zero mean and standard deviation, $\sigma$, then the expectation of the interferometric visibility is $\langle V\rangle=V e^{-\sigma^{2} / 2}$, where $V$ is the true visibility. See Chapter 13 of Thompson et al. (2001) for a derivation.
}

as the data were acquired under marginal weather conditions; we therefore remove these data also from our model fit. Fitting the centimeter-band data at $\lesssim 10 \mathrm{GHz}$ together with the ALMA observations, we find $\nu_{\mathrm{b}}=9.4 \pm 4.4 \mathrm{GHz}$ and $F_{\nu, \mathrm{pk}}=0.49 \pm$ $0.03 \mathrm{mJy}$ at $\approx 24.5$ days. The constancy of the peak flux density from 8.5 to 24.5 days identifies this break as $\nu_{\mathrm{m}}$ and confirms the circumburst medium as an ISM environment, for which we expect $F_{\nu, \mathrm{m}} \propto t^{0}$; the observed decline rate of this frequency, $\alpha_{\nu \text {,peak }}=-2.2 \pm 0.5$, is also consistent at $1.4 \sigma$ with the expectation of $\alpha_{\nu_{\mathrm{m}}}=-1.5$.

Projecting this frequency back to the optical bands at earlier times with $\alpha_{\nu}=-1.5$, we expect the break to have crossed $R$ band at $\approx 3 \times 10^{-2}$ days. Clear filter observations calibrated to $R$ band from Terksol at 0.29 days yield $f_{\nu, \mathrm{R}}=0.56 \pm 0.01$ mJy (Mazaeva et al. 2016), in excellent agreement with $\nu_{\mathrm{m}} \approx \nu_{\mathrm{opt}}$ at this time. This is further consistent with the subsequent decline rate and spectral index in the optical bands (Section 3.1), confirming the optical emission at $\gtrsim 3 \times$ $10^{-2}$ days and radio observations at $\gtrsim 8.5$ days as synchrotron emission from the FS. In the slow-cooling regime, the afterglow peak flux density is given by

$$
\begin{aligned}
F_{\nu} & =9.93(p+0.14)(1+z) \epsilon_{\mathrm{B}}^{1 / 2} n_{0}^{1 / 2} E_{\mathrm{K}, \text { iso }, 52} d_{\mathrm{L}, 28}^{-2} \mathrm{mJy} \\
& \sim 50 \mathrm{mJy}\left(\epsilon_{\mathrm{B},-2} n_{0}\right)^{1 / 2}\left(\frac{1-\eta_{\mathrm{rad}}}{\eta_{\mathrm{rad}}}\right) E_{\gamma, \text { iso }}
\end{aligned}
$$

for GRB $161219 \mathrm{~B}$ and $p \approx 2$, where $\eta_{\text {rad }}$ is the radiative efficiency. Taking $F_{\nu, \mathrm{m}} \approx 0.5 \mathrm{mJy}$ and assuming $\eta_{\mathrm{rad}} \approx 10 \%$, we find $n_{0} \approx 6 \times 10^{-4} \epsilon_{\mathrm{B},-2}^{-1} \mathrm{~cm}^{-3}$, indicating a low-density environment.

The ALMA light curve can be fit with a single power law with $\alpha_{1}=-0.52 \pm 0.02$ from the first observation at 1.3 days to the fourth epoch at $\approx 24.5$ days. This is shallower than the expected decline rate of FS emission and is best described as a combination of two emitting components declining at different rates (Section 6). This best-fit power law overpredicts the flux density at the fifth epoch at 78.2 days by a factor of $\approx 3$, which suggests that a jet break has occurred between 24.5 and 78.2 days, as indicated by the X-ray observations (Section 3.1). 
The radio SED fades at all frequencies between 24.5 and 79.0 days, and the best-fit broken power-law model at 79.0 days yields $\nu_{\mathrm{b}}=2.4 \pm 0.8 \mathrm{GHz}$ with flux density $F_{\nu \text {,pk }}=0.20 \pm 0.02 \mathrm{mJy}$ (fixing the same parameters as at 24.5 days). The drop in peak flux further indicates that a jet break has taken place between 24.5 and 79.0 days. The SED in the last epoch at 159.5 days can be fit either with a broken power law with $\beta_{1}=1 / 3$ (fixed), $\beta_{2} \approx-0.5 \quad$ (fixed), $\nu_{\mathrm{b}}=7.9 \pm 1.5 \mathrm{GHz}$, and $F_{\nu, \mathrm{pk}}=0.12 \pm 0.01 \mathrm{mJy}$ or as a single power law with $\beta=-0.8 \pm 0.1$. An increase in the break frequency $\nu_{\mathrm{b}}$ with time is physically implausible, and it is possible that the lowest-frequency observations in this epoch have contributions from the host galaxy. We discuss this epoch further in Section 7.6.

To summarize, the optical and X-ray light curves require a constant-density environment with $p \approx 2$. The multiband $\mathrm{X}$-ray through radio observations of the afterglow are consistent with a slow-cooling FS $\left(\nu_{\mathrm{m}}<\nu_{\mathrm{c}}\right)$ in an ISM environment with $\nu_{\mathrm{m}} \approx \nu_{\mathrm{opt}}$ at $\approx 3 \times 10^{-3}$ days, while the NIR to X-ray SED indicates $\nu_{\mathrm{c}} \approx \nu_{\mathrm{X}}$ for the duration of the X-ray observations. The UV spectral slope is marginally steeper than that in the optical bands, indicating possible extinction in the host galaxy. The peak flux density of the FS is $F_{\nu, \mathrm{m}} \approx 0.5 \mathrm{mJy}$, implying that the optical and $\mathrm{X}$-ray light curves prior to $\approx 3 \times 10^{-2}$ days and radio SEDs prior to 8.5 days are dominated by emission from a separate mechanism.

\section{Multiwavelength Modeling}

Our preliminary considerations described above indicate that the X-ray light curve after $\approx 0.1$ days, the UV, optical, and NIR data at $1.3 \times 10^{-2}-2$ days and at $>50$ days, and the radio SEDs at $\gtrsim 8.5$ days can be understood in the context of synchrotron radiation from an FS propagating into a constant-density ISM environment. We derive the parameters of this shock using the smoothly connected power-law synchrotron spectra described by Granot \& Sari (2002) modified using the weighting schemes presented in Laskar et al. (2014), including the effects of inverse Compton cooling (Sari \& Esin 2001; Laskar et al. 2015). The free parameters in this model are the total isotropic-equivalent kinetic energy $\left(E_{\mathrm{K} \text {,iso }}\right)$, the circumburst density $\left(n_{0}\right)$, the fraction of shock energy imparted to relativistic electrons $\left(\epsilon_{\mathrm{e}}\right)$, the fraction imparted to magnetic fields $\left(\epsilon_{\mathrm{B}}\right)$, and the index of the electron energy spectrum $(p)$. We incorporate the effects of collimation using the jet break time $\left(t_{\text {jet }}\right)$ as an additional free parameter (Rhoads 1999; Sari et al. 1999; Chevalier \& Li 2000; Laskar et al. 2014).

We include the contribution of the SN using a template constructed from the extinction-corrected spectra of SN 1998bw, performing $K$-corrections for the UV, optical, and NIR bands (Patat et al. 2001; Levan et al. 2005). The template is scaled by three additional parameters: the relative peak time $\left(\delta t_{\mathrm{sn}}\right)$, a temporal stretching factor $\left(\Upsilon_{\mathrm{sn}}\right)$, and a flux density scaling $\left(\Xi_{\mathrm{sn}}\right)$ using

$$
\begin{aligned}
t_{\mathrm{obs}} & =\Upsilon_{\mathrm{sn}} t_{0}+\delta t_{\mathrm{sn}}, \\
f_{\nu, \mathrm{obs}}\left(t_{\mathrm{obs}}\right) & =\Xi_{\mathrm{sn}} f_{\nu, 0},
\end{aligned}
$$

where $f_{0}\left(t_{0}\right)$ is the $\mathrm{SN} 1998 \mathrm{bw}$ template scaled to the redshift of GRB 161219B. Thus, $\Xi_{\text {sn }}$ measures the intrinsic luminosity ratio of $\mathrm{SN} 2016 \mathrm{jca}$ to $\mathrm{SN} 1998 \mathrm{bw}$, while $\delta t_{\mathrm{sn}} /(1+z)$ represents the rest-frame delay of the $\mathrm{SN} 2016 \mathrm{jca}$ peak relative to that of SN $1998 \mathrm{bw}$.

We model the extinction in the host galaxy using the SMC extinction curve (Pei 1992; Laskar et al. 2014) and include contributions from the host galaxy at all bands that exhibit a flattening at $\gtrsim 50$ days. Since the observations presented in Cano et al. (2017a) are measured using a 2!"2 aperture, the contribution of the host galaxy to their photometry is different from that in our observations. The largest differences appear in the $J H K$ bands, and we therefore keep the $J H K$ bands from the two sets of observations separate in our analysis. We include the pre-explosion griz Pan-STARRS1 photometry of the host (in the same $2 . ! 2$ aperture) as additional data points at the fiducial time of 200 days, at which time the contribution of the SN and afterglow is minimal, allowing us to constrain the contribution of the host to the light curve in all UV, optical, and NIR bands.

To efficiently sample parameter space and fully characterize the joint posterior density of the free parameters in our model, we carry out a Markov chain Monte Carlo (MCMC) analysis using the Python-based code EMCEE (Foreman-Mackey et al. 2013) following the procedure described in Laskar et al. (2014) and Laskar et al. (2015). We assume uninformative, uniform priors on all free parameters. Priors for all scale parameters $\left(\epsilon_{\mathrm{e}}\right.$, $\epsilon_{\mathrm{B}}, n_{0}, E_{\mathrm{K} \text {,iso }}, t_{\text {jet }}$, and $\left.\Upsilon_{\mathrm{SN}}\right)$ are uniform in logarithmic space (Jeffreys 1946). We initialized 128 Markov chains with parameters clustered around their best-fit values (with a 3\% dispersion). After discarding samples prior to the stabilization of the average likelihood across chains as "burn-in," we obtained $10^{4}$ samples from the posterior. Further details about our MCMC analysis method, convergence tests, and quantile and summary statistic calculation are available in Laskar et al. (2014). To account for calibration offsets in UV/optical/NIR data from different observatories, as well as potential systematic intrinsic flux calibration uncertainties, we impose an uncertainty floor of $10 \%$ prior to fitting with our modeling software.

\subsection{Afterglow}

In confirmation of the basic analysis presented in Section 3 , we find that an ISM model with $p \approx 2.08$ describes the data well. Our highest-likelihood (best-fit) model has $\nu_{\mathrm{m}} \approx 4 \times$ $10^{12} \mathrm{~Hz}<\nu_{\mathrm{opt}}$ and $\nu_{\mathrm{c}} \approx 2 \times 10^{17} \mathrm{~Hz} \approx \nu_{\mathrm{X}}$ at 1 day, as inferred from the optical and X-ray light curves. The extrapolated peak flux density is $F_{\nu, \mathrm{m}} \approx 1 \mathrm{mJy}$. The peak of the rounded spectrum $^{33}$ is then $F_{\nu, \mathrm{pk}}=2^{-1 / y} F_{\nu, \mathrm{m}} \approx 0.5 \mathrm{mJy}$, consistent with the radio SEDs and the optical observations at $\approx 3 \times 10^{-2}$ days (Section 3.2). The afterglow remains in the slow-cooling regime for the duration of the observations.

This model also requires a jet break at $t_{\text {jet }} \approx 32$ days, corresponding to an opening angle of $\approx 13^{\circ}$ (Sari et al. 1999). The resulting beaming-corrected kinetic and $\gamma$-ray energies are $E_{\mathrm{K}} \approx 1.3 \times 10^{50} \mathrm{erg}$ and $E_{\gamma} \approx 4.9 \times 10^{48} \mathrm{erg}$, respectively. The corresponding radiative efficiency is extremely low, $\eta \approx 4 \%$ (independent of the beaming angle). We discuss this further in Section 6. This break time is later than derived from fitting the X-ray light curve alone (Section 3.1), owing to the steeper post-break decline rate in the physical model compared to the simple power-law fits of the X-ray light curve. The resulting model matches the $\mathrm{X}$-ray data at $\gtrsim 0.1$ days fairly well (Figure 5). We note that the time of the jet break is partly driven by the ALMA light curve, which declines as $\alpha=-1.5 \pm 0.3$ between 24.5 and 78.2 days, steeper than the expected value of $\approx-0.8$ for the ordering $\nu_{\mathrm{m}}<\nu_{\mathrm{ALMA}}<\nu_{\mathrm{c}}$

\footnotetext{
${ }^{33}$ Here $y=1.84-0.40 p \approx 1.0$ is the smoothness of the $\nu_{\mathrm{m}}$ break.
} 

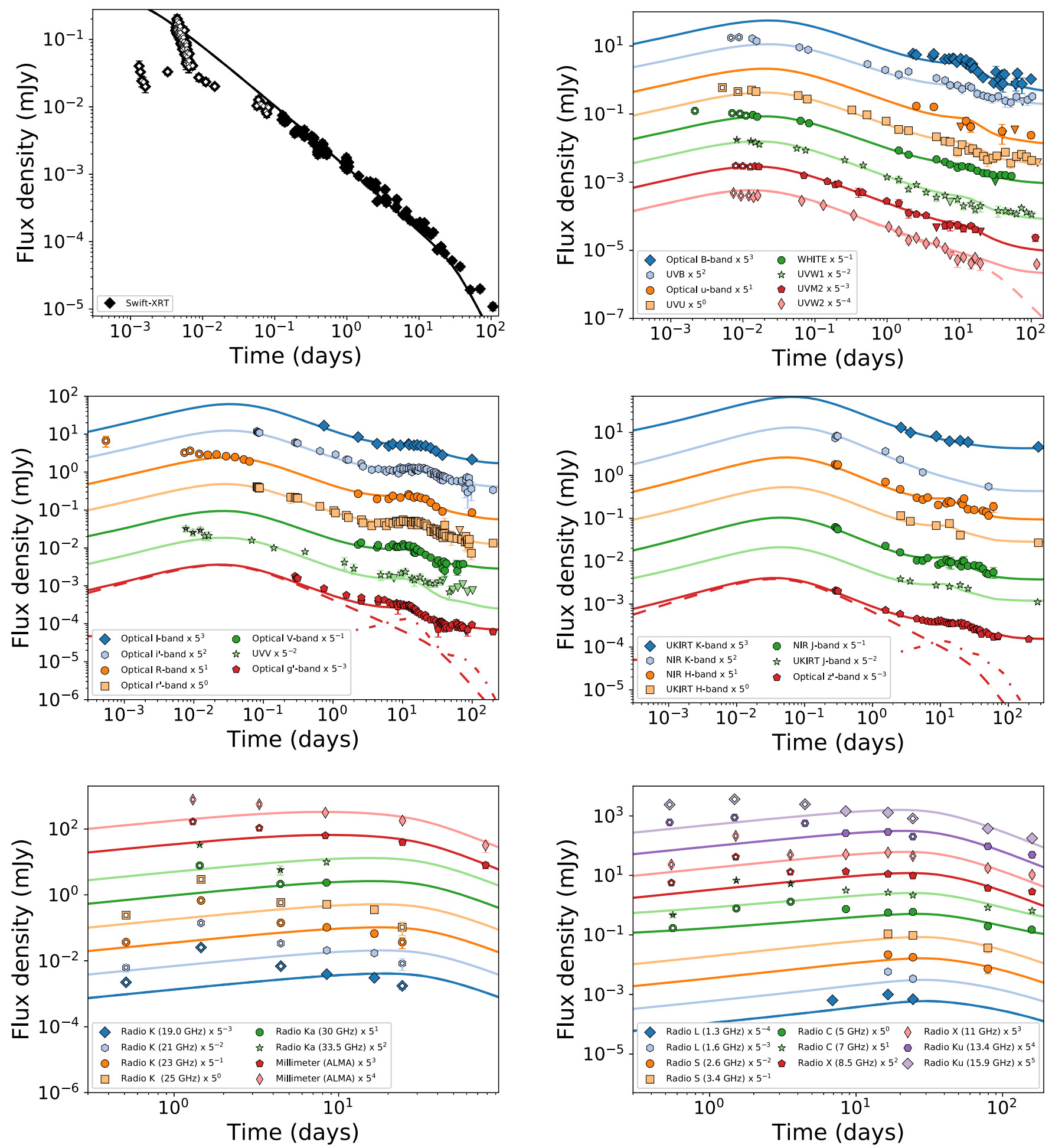

Figure 5. X-ray (top left), UV (top right), optical (center left), NIR (center right), and radio (bottom) light curves of GRB 161219B/SN 2016jca, together with an FS ISM model including contributions from the SN light (solid lines). We show a decomposition of the Swift/w2-band, optical $g^{\prime}$-band, and optical $z^{\prime}$-band light curves into FS (dashed) and SN (dot-dashed) components. Data represented by open symbols are not included in the model fit. The JHK photometry from GROND, NOT, and GTC was reported in a $2.2^{\prime \prime}$ aperture and does not include the full light of the host; these bands are therefore treated separately from the UKIRT photometry, which does include all contributions from the host (Section 4). This FS-only model overpredicts the X-ray data at $\lesssim 0.1$ days and underpredicts the optical observations at $\lesssim 3 \times 10^{-3}$ days, as well as the radio observations at $\lesssim 8.5$ days; both deficiencies are overcome in the refreshed RS model presented in Figure 10 .

and a spherical, adiabatic shock, as also discussed in Section 3.2. The resulting model light curve matches the ALMA flux density in the final three epochs but underpredicts the millimeter- and centimeter-band observations before $\approx 8.5$ days (Figure 6 ). The parameters for the best-fit model, together with the median and $68 \%$ credible intervals from the 

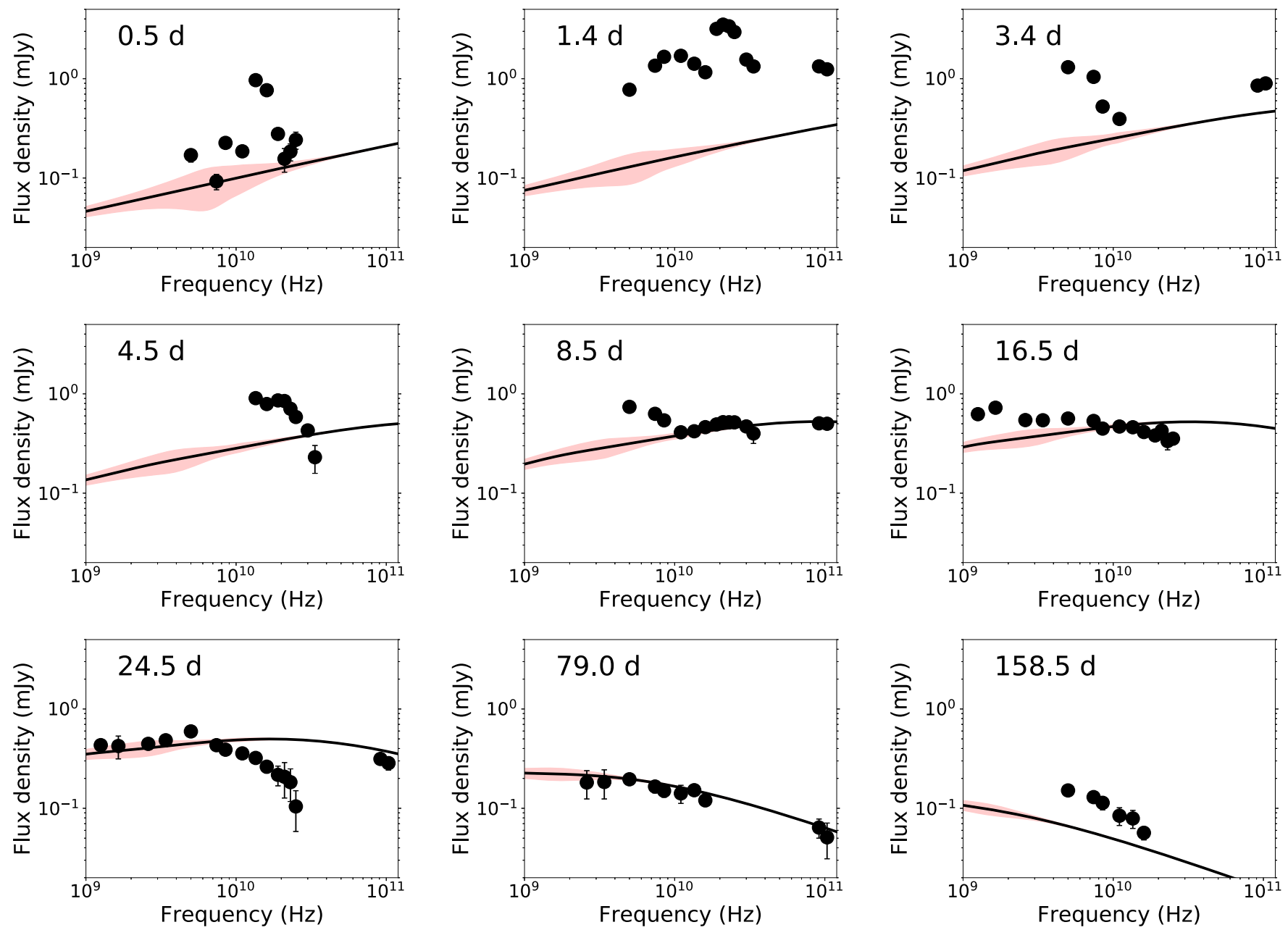

Figure 6. Multifrequency centimeter-band (VLA) and millimeter-band (ALMA) SEDs of the afterglow of GRB 161219B, together with an FS ISM model (solid lines; Section 4). The red shaded regions represent the expected variability due to scintillation. This FS-only model underpredicts the radio observations at $\lesssim 8.5$ days and requires an additional component (Section 6 and Figure 12).

MCMC analysis, are provided in Table 8, and histograms of the marginalized posterior density are presented Figure 7 . We note that $\nu_{\mathrm{a}}$ is not constrained by the data, resulting in some degeneracies between the physical parameters (Figure 8).

\subsection{Supernova}

The SN (SN 2016jca) associated with this burst has previously been studied by Ashall et al. (2017) and Cano et al. (2017a), who consider both magnetar and radioactive decay models for powering the SN light curve. Ashall et al. (2017) argue for the magnetar model with an ejecta mass of $M_{\mathrm{sn}, \mathrm{ej}} \approx 8 M_{\odot}$, despite the high isotropic-equivalent ejecta kinetic energy required, $E_{\mathrm{sn}, \mathrm{K} \text {,iso }} \approx 5.4 \times 10^{54} \mathrm{erg}$. Their afterglow model used to derive the $\mathrm{SN}$ light curve requires $p<2$, while the large jet opening angle they infer, $\theta_{\text {jet }} \approx 40^{\circ}$, is based on an assumed circumburst density of $n_{0} \approx 1 \mathrm{~cm}^{-3}$, over 3 orders of magnitude larger than the value obtained here from multiwavelength modeling. Cano et al. (2017a) derive a lower ejecta mass, $M_{\mathrm{sn}, \mathrm{ej}}=5.8 \pm 0.3 M_{\odot}$, and a similar ejecta kinetic energy, $E_{\mathrm{sn}, \mathrm{K} \text {,iso }}=(5.1 \pm 0.8) \times 10^{54} \mathrm{erg}$. Under the assumption that the SN light curve is powered by radioactive decay of ${ }^{51} \mathrm{Ni}$, they find a nickel mass of $M_{\mathrm{Ni}}=0.22 \pm 0.08 M_{\odot}$ and $\gamma$-ray opacity $\kappa_{\gamma} \approx 0.034 \mathrm{~cm}^{2} \mathrm{~g}^{-1}$. Our method, which assumes the same color evolution as the template, yields a stretch factor of $\Upsilon_{\mathrm{sn}} \approx 0.8$ and a flux scale factor $^{34}$ of $\Xi_{\mathrm{sn}} \approx 0.8$, within $\approx 1 \sigma$ of the correlation between these parameters derived by Cano (2014). Whereas our method does not allow us to derive specific physical parameters of SN $2016 \mathrm{jca}$, our results are broadly consistent with those of Cano et al. (2017a), who find (frequency-dependent) stretch factors of $\Upsilon_{\mathrm{sn}} \approx 0.6-0.9$ and $\Xi_{\mathrm{sn}} \approx 0.7-0.8$.

\subsection{Host Galaxy}

We derive an SED for the host galaxy using five of the six narrowband UVOT filters, ${ }^{35}$ together with the pre-explosion PS1 grizy host photometry (Cano et al. 2017a) and our JHK data (Figure 9). We fit a set of galaxy templates from Bruzual \& Charlot (2003) using FAST (Kriek et al. 2009), assuming an exponentially declining star formation history ( $\tau$-model), a Chabrier (2003) initial mass function, and a stellar metallicity

\footnotetext{
34 These correspond to the parameters $k$ and $s$ of Cano (2014), respectively. We use different symbols in this work to avoid confusion with the ejecta Lorentz factor distribution (Equation (7)) and the circumburst medium density profile index.

35 Photometry in the UVOT $v$ band is most significantly affected by diffracted light from the nearby star and is less reliable than in the other bands at late times. We therefore exclude this band from the SED fit.
} 
Table 8

Results of Multiwavelength Modeling

\begin{tabular}{lll}
\hline \hline Parameter & \multicolumn{1}{c}{ Best Fit } & MCMC \\
\hline Forward Shock & & \\
\hline$p$ & 2.08 & $2.079_{-0.006}^{+0.009}$ \\
$\epsilon_{\mathrm{e}}$ & 0.93 & $0.89_{-0.07}^{+0.05}$ \\
$\epsilon_{\mathrm{B}}$ & $5.1 \times 10^{-2}$ & $\left(5.8_{-3.0}^{+5.4}\right) \times 10^{-2}$ \\
$n_{0}\left(\mathrm{~cm}^{-3}\right)$ & $3.6 \times 10^{-4}$ & $\left(3.2_{-1.2}^{+1.4}\right) \times 10^{-4}$ \\
$E_{\mathrm{K}, \text { iso,52 }}(\mathrm{erg})$ & 0.47 & $0.46_{-0.09}^{+0.14}$ \\
$t_{\text {jet }}(\mathrm{days})$ & 31.5 & $33.0_{-1.4}^{+1.5}$ \\
$\theta_{\text {jet }}(\mathrm{deg})$ & 13.5 & $13.44 \pm 0.35$ \\
$A_{\mathrm{V}}(\mathrm{mag})$ & $3.0 \times 10^{-2}$ & $\left(2.1_{-2.1}^{+2.0}\right) \times 10^{-2}$ \\
$E_{\mathrm{K}}(\mathrm{erg})$ & $1.3 \times 10^{50}$ & $\left(1.27_{-0.25}^{+0.36}\right) \times 10^{50}$ \\
\hline
\end{tabular}

Prompt Emission

\begin{tabular}{lll}
\hline$E_{\gamma, \text { iso }}$ & $(1.8 \pm 0.4) \times 10^{50}$ & $\ldots$ \\
$E_{\gamma}(\mathrm{erg})$ & $4.9 \times 10^{48}$ & $(4.9 \pm 1.9) \times 10^{48}$ \\
$\eta_{\text {rad }}$ & $3.7 \%$ & $\ldots$ \\
\hline \multicolumn{2}{c}{$S N$ 2016jca } \\
\hline$\delta t_{\text {sn,peak }}$ (days) & -3.7 & $-4.10_{-0.96}^{+0.80}$ \\
$\Upsilon$ & 0.83 & $0.84 \pm 0.04$ \\
$\Xi_{\mathrm{f}}$ & 0.73 & $0.76 \pm 0.02$ \\
\hline
\end{tabular}

\begin{tabular}{|c|c|c|}
\hline \multicolumn{3}{|c|}{ Mean Host Contribution ( $\mu J y)$} \\
\hline$u w 2$ & 1.64 & $1.57 \pm 0.34$ \\
\hline$u m 2$ & 1.48 & $1.12_{-0.51}^{+0.30}$ \\
\hline$u w 1$ & 2.35 & $2.39 \pm 0.27$ \\
\hline$u w h$ & 5.03 & $4.90 \pm 0.35$ \\
\hline$u v u$ & 3.61 & $3.26 \pm 0.39$ \\
\hline$u^{\prime}$ & 2.92 & $2.77_{-0.81}^{+0.59}$ \\
\hline$u v b$ & 8.35 & $8.03_{-0.69}^{+0.38}$ \\
\hline$B$ & 3.89 & $3.64 \pm 0.25$ \\
\hline$g^{\prime}$ & 9.58 & $9.28 \pm 0.27$ \\
\hline$u v v$ & 6.61 & $5.32 \pm 0.88$ \\
\hline$V$ & 15.3 & $14.0 \pm 0.8$ \\
\hline$r^{\prime}$ & 13.2 & $12.8 \pm 0.6$ \\
\hline$R$ & 11.9 & $10.6 \pm 1.0$ \\
\hline$i^{\prime}$ & 17.1 & $16.4 \pm 0.6$ \\
\hline$I$ & 14.1 & $13.4 \pm 1.0$ \\
\hline$z^{\prime}$ & 20.2 & $19.6 \pm 0.65$ \\
\hline$U K I R T-J$ & 30.6 & $31.5 \pm 2.1$ \\
\hline$J$ & 19.2 & $17.3 \pm 1.2$ \\
\hline$U K I R T-H$ & 28.7 & $29.3 \pm 2.5$ \\
\hline$H$ & 19.1 & $18.5 \pm 1.2$ \\
\hline$K$ & 17.3 & $18.4 \pm 2.0$ \\
\hline$U K I R T-K$ & 34.5 & $37.5 \pm 2.5$ \\
\hline $5 \mathrm{GHz}$ & 60.2 & $52.0 \pm 16.4$ \\
\hline $7.4 \mathrm{GHz}$ & 42.0 & $41.2 \pm 13.2$ \\
\hline
\end{tabular}

of $Z=0.008$ ( 0.4 solar, corresponding to the value for the host obtained from $\mathrm{H} \alpha$ and emission-line diagnostics; Cano et al. 2017a). Whereas the extinction and $\tau$ are particularly susceptible to systematic photometric uncertainties in the Swift photometry and are poorly constrained by the weak UV detections of the host, the stellar mass is well determined, $\log \left(M_{*} / M_{\odot}\right)=8.92_{-0.02}^{+0.04}$. We derive a stellar population age, $\log t_{0}=9.0_{-0.1}^{+0.2}, \quad \tau \approx 0.3 \mathrm{Gyr}$, rest-frame extinction, $A_{\mathrm{V}}=$ $0.6_{-0.6}^{+0.2} \mathrm{mag}$, and current star formation rate $\mathrm{SFR}=$ $0.19_{-0.16}^{+0.02} M_{\odot} \mathrm{yr}^{-1}$. These values are similar to those derived
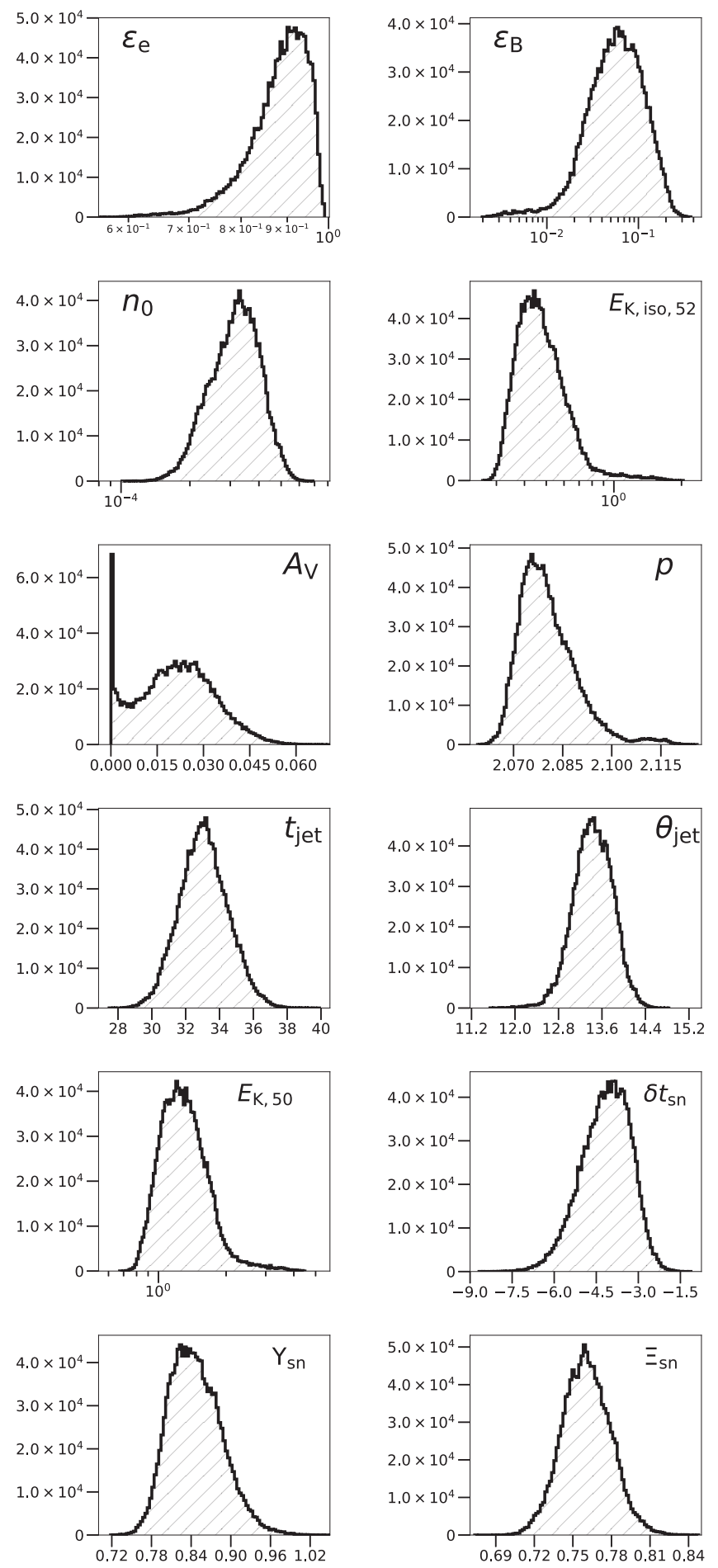

Figure 7. Posterior probability density functions for the physical parameters for GRB 161219B and the light curve of SN 2016jca.

by Cano et al. (2017a) using the PS1 photometry alone. The derived stellar mass is comparable to the mean stellar mass of GRB hosts at $z \lesssim 1\left(\log \left(M_{*} / M_{\odot}\right)=9.25_{-0.23}^{+0.19}\right.$; Levesque et al. 2010). On the other hand, the specific SFR, $\log \left[\mathrm{sSFR} / \mathrm{Gyr}^{-1}\right] \approx-0.65$, appears to be an order of magnitude lower than the median SSFR of GRB hosts at $z \lesssim 1\left(\log \left[\mathrm{sSFR} / \mathrm{Gyr}^{-1}\right] \approx 0.3\right.$; Levesque et al. 2010). The possibility that the GRB occurred in an extreme star-forming 

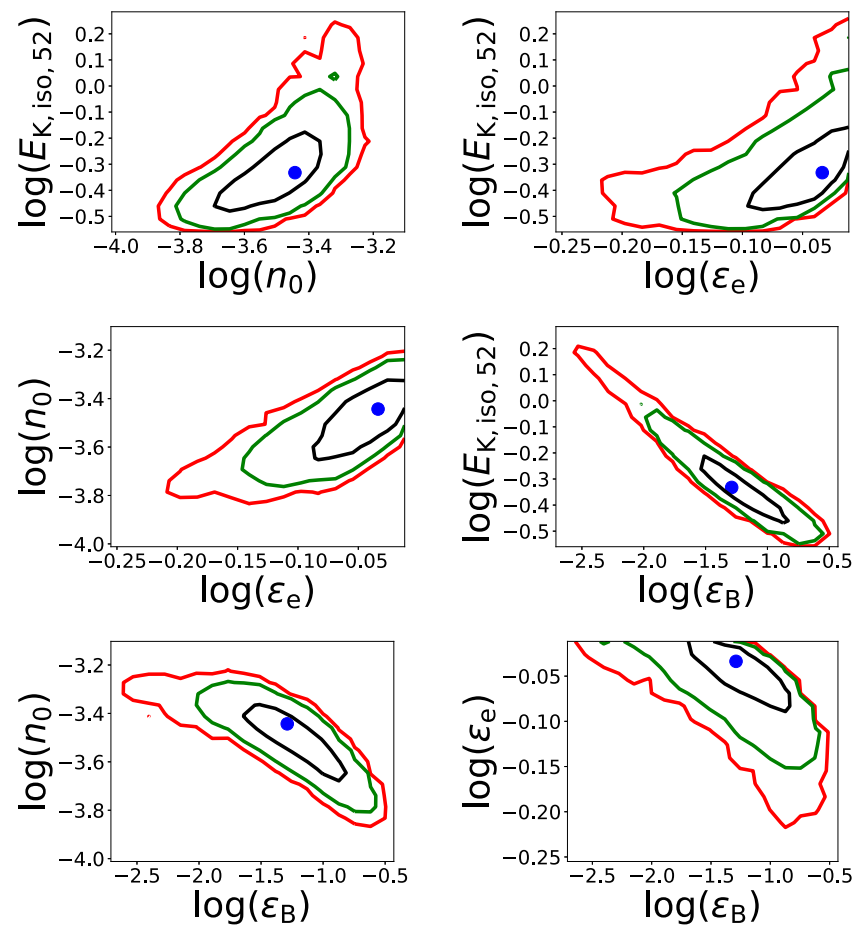

Figure 8. $1 \sigma$ (red), $2 \sigma$ (green), and $3 \sigma$ (black) contours for correlations between the physical parameters $E_{\mathrm{K} \text {,iso }}, n_{0}, \epsilon_{\mathrm{e}}$, and $\epsilon_{\mathrm{B}}$ for GRB $161219 \mathrm{~B}$ from Monte Carlo simulations. The blue dot indicates the highest-likelihood model. We have restricted $\epsilon_{\mathrm{e}}+\epsilon_{\mathrm{B}}<1$. The complete set of 66 correlation plots is available as a figure set online.

(The complete figure set (66 images) is available.)

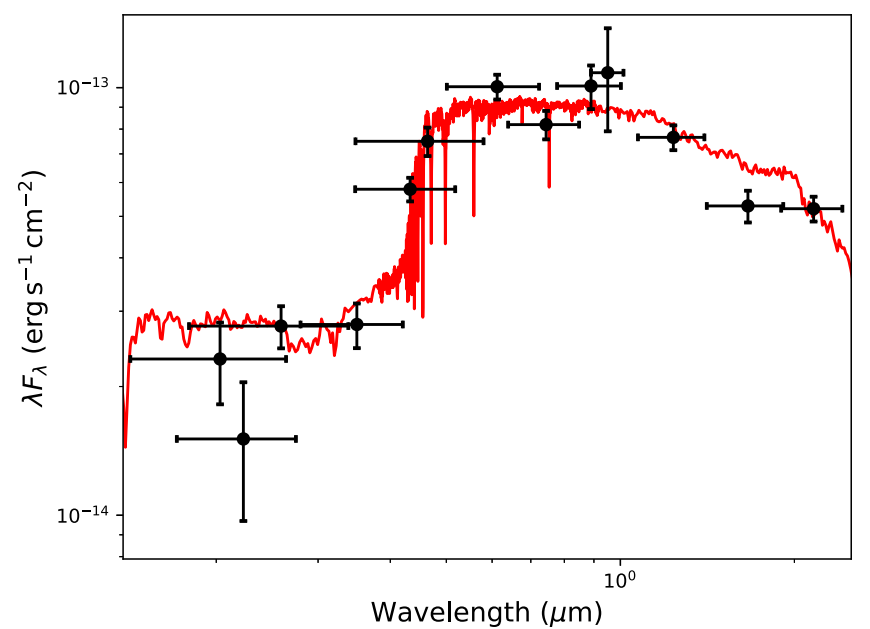

Figure 9. SED of the host of GRB 161219B derived from multiwavelength modeling (Section 4), together with a best-fit model from Bruzual \& Charlot (2003).

region within an otherwise low sSFR host is disfavored by $H S T$ spectroscopy of the SN site (Cano et al. 2017a). Dust extinction may impact the derived SFR by extinguishing the light from young stars, especially in an edge-on system like the host of GRB 161219B; however, we derive a low extinction from afterglow modeling, consistent with the host SED fits. Since long-duration GRBs are typically associated with regions of the most intense star formation in their hosts (Bloom et al. 2002; Fruchter et al. 2006; Svensson et al. 2010; Blanchard et al. 2016), the lack of evidence for strong star formation activity at the GRB site is puzzling.

\section{Energy Injection}

The optical and X-ray light curves exhibit an unusual achromatic break at $\approx 0.1$ days, which cannot be explained in the standard synchrotron framework. Furthermore, the model described in Section 4 overpredicts the X-ray light curve before $\approx 0.1$ days (Figure 5 ). One of the simplest means to obtain a flatter light curve at earlier times is through the injection of energy into the FS due to extended activity of the central engine, the deceleration of a Poynting-flux-dominated outflow, or stratified ejecta with additional energy available at lower Lorentz factors (Dai \& Lu 1998; Rees \& Meszaros 1998; Kumar \& Panaitescu 2000; Sari \& Mészáros 2000; Zhang \& Mészáros 2001, 2002; Granot \& Kumar 2006; Zhang et al. 2006; Dall'Osso et al. 2011; Uhm et al. 2012). The effect on the FS is a gradual increase in the effective shock energy with time, $E \propto t^{m} \propto t^{1-q}$, where $-q \equiv m-1$ is the power-law index of the injection luminosity, $L \propto t^{-q}$ (Zhang et al. 2006). For energy injection due to accumulation from a distribution of ejecta Lorentz factors, the corresponding ejecta energy distribution is given by $E(>\Gamma) \propto \Gamma^{1-s}$, with

$$
s=\frac{(7 m+3)-k(2 m+1)}{(3-k)-m},
$$

where the external density profile as a function of radius, $R$, is assumed to follow the general ${ }^{36}$ power-law form, $\rho=A R^{-k}$. During this process, the FS Lorentz factor, shock radius, and post-shock magnetic field all evolve more slowly than the standard relativistic solution (Blandford \& McKee 1976; Sari \& Mészáros 2000; Zhang et al. 2006),

$$
\begin{aligned}
& \frac{\partial \ln \Gamma}{\partial \ln t}=-\frac{q+2-k}{2(4-k)}=-\frac{3-k}{7+s-k}, \\
& \frac{\partial \ln R}{\partial \ln t}=\frac{2-q}{4-k}=\frac{1+s}{7+s-2 k}, \\
& \frac{\partial \ln B}{\partial \ln t}=-\frac{q+k+2-k q}{2(4-k)}=-\frac{6+k s-k}{2(7+s-2 k)},
\end{aligned}
$$

with $s$ and $q$ related by

$$
\begin{aligned}
& s=\frac{10-3 k-7 q+2 k q}{2+q-k}, \\
& q=\frac{10-2 s-3 k+k s}{7+s-2 k} .
\end{aligned}
$$

The standard hydrodynamic evolution in the absence of energy injection can be recovered by setting $m=0, s=1$, or $q=1$ in the above expressions (e.g., Gao et al. 2013).

In our best-fit model, $\nu_{\mathrm{X}}<\nu_{\mathrm{c}}$ at $\lesssim 0.1$ days, whereupon $m=\left(4 \alpha_{\mathrm{X}}+3 p-3\right) /(p+3)=0.35 \pm 0.09$ using $\alpha_{\mathrm{X}}=$ $-0.37 \pm 0.09$ (Section 3.1), which implies $s \approx 2$ for $k=0$ (Equation (7)) in the massive ejecta model. No theoretical models yet exist of the expected distribution of ejecta Lorentz factors, and in fact the distribution need not follow a power law. However, our observations of energy injection in this

\footnotetext{
36 We keep the discussion here general for completeness, and we specialize to the ISM $(k=0)$ case later.
} 
event add to the growing collection of a measurement of $s$ in GRB jets (Laskar et al. 2015).

We note that the FS cooling frequency $\nu_{\mathrm{c}, \mathrm{f}} \propto$ $E^{-1 / 2} t^{-1 / 2} \propto t^{-(m+1) / 2} \sim t^{-0.65}$. Thus, in our model $\nu_{\mathrm{c}, \mathrm{f}}$ evolves from $\approx 3 \times 10^{18} \mathrm{~Hz}$ to $\approx 7 \times 10^{17} \mathrm{~Hz}$ between the end of the flare at $\approx 0.01$ days and the end of energy injection at $\approx 0.1$ days. The presence of $\nu_{\mathrm{c}, \mathrm{f}}$ within the Swift X-ray band explains the observed X-ray spectral index, $\beta_{\mathrm{X}} \approx-0.86$, which is intermediate between $(1-p) / 2 \approx-0.54$ and $-p / 2 \approx$ -1.04 .

Since the peak flux density is $\approx 0.5 \mathrm{mJy}$ and $f_{\nu, \mathrm{m}}$ is constant in an ISM environment, a measured flux density greater than this value at any frequency and time cannot be explained by FS emission. Thus, as we previously argued, the optical light curve before $\approx 3 \times 10^{-2}$ days and the radio SEDs before $\approx 8.5$ days must be dominated by a distinct emission component. Whereas energy injection can explain the relatively flat X-ray light curve before $\approx 0.1$ days, adding this to our model further worsens the fit to the optical light curves at that time. We address both concerns in the next section.

\section{Reverse Shock}

During the process of energy injection, an RS mediates the transfer of energy from the ejecta into the FS. This RS, which is Newtonian or mildly relativistic, propagates for the period of the injection and (by definition) crosses the ejecta at the time $\left(t_{\mathrm{E}}\right)$ when energy injection terminates (Rees \& Meszaros 1998; Kumar \& Piran 2000; Zhang et al. 2003). Such a "long-lasting" RS propagating into the ejecta released during the GRB may produce detectable synchrotron radiation (Sari \& Mészáros 2000; Uhm 2011). We now show that such an RS can reproduce the observed excess in both the optical light curves at $\lesssim 3 \times 10^{-2}$ days and the radio SEDs at $\lesssim 8.5$ days, beginning first with the theoretical model (Section 6.1), followed by the results from our data (Section 6.2) and consistency checks between theory and observations (Section 6.3).

\subsection{Energy Injection RS-Theoretical Prescription}

A detailed calculation of the hydrodynamics of the double shock system requires numerical simulations or semianalytic modeling. Here we follow previous analytic work by Sari \& Mészáros (2000) and Uhm (2011) and make the simplifying assumption that the pressure behind the RS is equal to that at the FS, $P \propto \Gamma^{2} \rho$ (however, see Uhm et al. 2012, for a discussion of situations where this assumption is relaxed). The characteristic frequency, cooling frequency, and peak flux density of the radiation from the RS and FS are then related during the shock crossing $\left(t<t_{\mathrm{E}}\right)$ by

$$
\begin{aligned}
\frac{\nu_{\mathrm{m}, \mathrm{r}}}{\nu_{\mathrm{m}, \mathrm{f}}} & \sim \Gamma^{-2} R_{\mathrm{B}} R_{\mathrm{e}}^{2}, \\
\frac{\nu_{\mathrm{c}, \mathrm{r}}}{\nu_{\mathrm{c}, \mathrm{f}}} & \sim R_{\mathrm{B}}^{-3}\left(\frac{1+Y_{\mathrm{f}}}{1+Y_{\mathrm{r}}}\right)^{2}, \\
\frac{F_{\nu, \mathrm{m}, \mathrm{r}}}{F_{\nu, \mathrm{m}, \mathrm{f}}} & \sim \Gamma R_{\mathrm{B}},
\end{aligned}
$$

where $\Gamma$ is the Lorentz factor of the FS, $R_{\mathrm{B}} \equiv\left(\epsilon_{\mathrm{B}, \mathrm{r}} / \epsilon_{\mathrm{B}, \mathrm{f}}\right)^{1 / 2}$ is the ejecta magnetization, $Y_{\mathrm{r}}$ and $Y_{\mathrm{f}}$ are the Compton $Y$-parameters for the $\mathrm{RS}$ and $\mathrm{FS}$, respectively, and $R_{\mathrm{e}} \equiv$ $\bar{\epsilon}_{\mathrm{e}, \mathrm{r}} / \bar{\epsilon}_{\mathrm{e}, \mathrm{f}}$, with $\bar{\epsilon}_{\mathrm{e}} \equiv(p-2) \epsilon_{\mathrm{e}} /(p-1)$ (Zhang et al. 2006).
We assume the same value of $p$ for both the RS and FS, so that $R_{\mathrm{e}}=\epsilon_{\mathrm{e}, \mathrm{r}} / \epsilon_{\mathrm{e}, \mathrm{f}}$. As for the FS, we assume that the microphysical parameters of the RS (and hence $R_{\mathrm{e}}$ and $R_{\mathrm{B}}$ ) remain constant with time. Thus, the RS spectral parameters are directly proportional to those of the FS during shock crossing:

$$
\begin{aligned}
\nu_{\mathrm{m}, \mathrm{r}} & \propto \Gamma^{-2} \nu_{\mathrm{m}, \mathrm{f}} \\
\nu_{\mathrm{c}, \mathrm{r}} & \propto \nu_{\mathrm{c}, \mathrm{f}}, \\
F_{\nu, \mathrm{m}, \mathrm{r}} & \propto \Gamma F_{\nu, \mathrm{m}, \mathrm{f} .}
\end{aligned}
$$

The number of electrons swept up by the FS (prior to the jet break) is given by $N_{\mathrm{e}, \mathrm{f}} \propto R^{3} \rho \propto R^{3-k}$. Since $\nu_{\mathrm{m}, \mathrm{f}} \propto \Gamma \gamma_{\mathrm{e}}^{2} B$, $\nu_{\mathrm{c}, \mathrm{f}} \propto \Gamma^{-1} B^{-3} t^{-2}$, and $F_{\nu, \mathrm{m}, \mathrm{f}} \propto N_{\mathrm{e}, \mathrm{f}} B \Gamma$, while the minimum Lorentz factor of accelerated electrons $\gamma_{\mathrm{e}} \propto \Gamma$, the spectral parameters of the FS at $t<t_{\mathrm{E}}$ are (Zhang et al. 2006)

$$
\begin{aligned}
\frac{\partial \ln \nu_{\mathrm{m}, \mathrm{f}}}{\partial \ln t} & =-\frac{q+2}{2}, \\
\frac{\partial \ln \nu_{\mathrm{c}, \mathrm{f}}}{\partial \ln t} & =\frac{(3 k-4)(2-q)}{2(4-k)}, \\
\frac{\partial \ln F_{\nu, \mathrm{m}, \mathrm{f}}}{\partial \ln t} & =\frac{3 k q-4 k-8 q+8}{2(4-k)} .
\end{aligned}
$$

These equations reduce to the standard results in the absence of energy injection $(q=1)$ and can also be recovered by setting $E \propto t^{m}$ in the expressions given by Granot \& Sari (2002). Combining Equations (11) and (12), the spectral parameters of the RS at $t<t_{\mathrm{E}}$ are

$$
\begin{aligned}
\frac{\partial \ln \nu_{\mathrm{m}, \mathrm{r}}}{\partial \ln t} & =-\frac{2 q-k q+4}{2(4-k)}, \\
\frac{\partial \ln \nu_{\mathrm{c}, \mathrm{r}}}{\partial \ln t} & =\frac{(3 k-4)(2-q)}{2(4-k)}, \\
\frac{\partial \ln F_{\nu, \mathrm{m}, \mathrm{r}}}{\partial \ln t} & =\frac{3(k q-k-3 q+2)}{2(4-k)},
\end{aligned}
$$

which yield the expressions of Sari \& Mészáros (2000) for $k=0$.

The evolution of the RS self-absorption frequency during energy injection is more complex and depends on the relative ordering of $\nu_{\mathrm{a}, \mathrm{r}}, \nu_{\mathrm{m}, \mathrm{r}}$, and $\nu_{\mathrm{c}, \mathrm{r}}$. When both the RS and FS are in the slow-cooling regime $\left(\nu_{\mathrm{m}, \mathrm{r}}<\nu_{\mathrm{c}, \mathrm{r}}\right.$ and $\left.\nu_{\mathrm{m}, \mathrm{f}}<\nu_{\mathrm{c}, \mathrm{f}}\right)$, we expect $\nu_{\mathrm{a}, \mathrm{r}} \propto \Gamma^{8 / 5} \nu_{\mathrm{a}, \mathrm{f}}$ at $t<t_{\mathrm{E}}$ (Sari \& Mészáros 2000), so that

$$
\frac{\partial \ln \nu_{\mathrm{m}, \mathrm{r}}}{\partial \ln t}=-\frac{8}{5}\left[\frac{q+2-k}{2(4-k)}\right]+\frac{\partial \ln \nu_{\mathrm{m}, \mathrm{f}}}{\partial \ln t},
$$

which equals $-\frac{q+2}{5}$ (slower than the evolution of $\nu_{\mathrm{m}, \mathrm{r}}$ ) for the ISM case. We later show (Section 6.2) that $\nu_{\mathrm{m}, \mathrm{r}} \approx \nu_{\mathrm{a}, \mathrm{r}}$ at $\approx 1.4$ days, so that $\nu_{\mathrm{a}, \mathrm{r}}$ does not affect the light curve at any observed frequency prior to the end of energy injection at $t_{\mathrm{E}}$. We therefore ignore self-absorption in the RS prior to $t_{\mathrm{E}}$.

After injection ends, the residual RS spectrum fades according to the standard RS prescription (Kobayashi 2000; Zou et al. 2005). The evolution of $\nu_{\mathrm{a}, \mathrm{r}}, \nu_{\mathrm{m}, \mathrm{r}}, \nu_{\mathrm{c}, \mathrm{r}}$, and $F_{\nu, \mathrm{m}, \mathrm{r}}$ at $t>t_{\mathrm{E}}$ depends on whether the RS was Newtonian or relativistic. For a relativistic RS, no additional parameters are necessary, while for a Newtonian RS, we follow Kobayashi \& Sari (2000) in parameterizing the evolution of the ejecta 
Lorentz factor as $\Gamma \propto R^{-g}$. Since the shocked ejecta lag the FS and the FS Lorentz factor evolves with radius as $t^{(3-k) / 2}$, we expect $g>(3-k) / 2$. On the other hand, the fluid Lorentz factor in the adiabatic Blandford \& McKee (1976) solution evolves as $\gamma_{\mathrm{f}} \propto t^{(2 k-7) / 2}$ (Wu et al. 2003); since a Newtonian RS does not decelerate the ejecta effectively, its Lorentz factor is expected to evolve with radius slower than the BlandfordMcKee solution. Thus, $(3-k) / 2 \leqslant g \leqslant(2 k-7) / 2$, or $3 / 2 \leqslant g \leqslant-7 / 2$ for the ISM environment and $1 / 2 \leqslant g \leqslant$ $3 / 2$ in the wind case.

Using numerical simulations, Kobayashi \& Sari (2000) found $g \approx 2$ for a standard Newtonian RS not associated with energy injection in the ISM environment and $g \approx 3$ for a relativistic RS. Recent observations of radio afterglows have constrained $g \approx 5$ for GRB 130427A (Laskar et al. 2013; Perley et al. 2014) in the wind environment (outside the canonical range) and $g \approx 2$ for GRB 160509A in an ISM environment (Laskar et al. 2016). We consider both the relativistic and Newtonian prescriptions for evolution at $t>t_{\mathrm{E}}$ in our analysis, discussing self-consistency in Section 6.3. Following the jet break, the evolution of $F_{\nu, \mathrm{m}, \mathrm{r}}$ steepens further by a factor of $\Gamma^{2}$ due to geometric effects (Rhoads 1999; de Colle et al. 2012; Granot \& Piran 2012; Laskar et al. 2016), and we include this in our modeling.

\subsection{Energy Injection $R S$-Observational Constraints}

A detailed study of spectro-temporal variability in the radio afterglow in our companion paper (ALB18) indicates the variability peaks at $10-30 \mathrm{GHz}$ at $t \lesssim 8.5$ days, but it is minimal at lower frequencies and in the ALMA bands (Figure 4). We therefore anchor our RS model to the LSC bands at centimeter wavelengths $(1 \sim 10 \mathrm{GHz})$ and to the ALMA bands at $\approx 100 \mathrm{GHz}$. From the observed centimeter- to millimeter-band SEDs (Figure 6), we require the RS spectral peak, $\nu_{\mathrm{m}, \mathrm{r}} \approx 10 \mathrm{GHz}$ at 1.4 days, with $F_{\nu, \mathrm{m}, \mathrm{r}} \approx 4 \mathrm{mJy}$. Since the flux density in the first epoch at $\approx 0.5$ days is $\lesssim 1 \mathrm{mJy}$ at all bands and the RS light curves rise below $\nu_{\mathrm{a}, \mathrm{r}}$ and fade below $\nu_{\mathrm{m}, \mathrm{r}}$, we expect $\nu_{\mathrm{a}, \mathrm{r}}$ to be located at $1-10 \mathrm{GHz}$ at 0.5 and 1.4 days to explain the observed brightening from between the first two epochs. Since the millimeter-band data are brighter than the prediction from the FS at both 1.4 and 3.4 days, the RS must contribute some flux at those frequencies, and hence $\nu_{\mathrm{c}, \mathrm{r}} \gtrsim 100 \mathrm{GHz}$ at 3.4 days. On the other hand, we require $\nu_{\mathrm{c}, \mathrm{r}} \lesssim \nu_{\mathrm{opt}}$ at $\approx 10^{-2}$ days so as to not overpredict the $\mathrm{UV} /$ optical light curves before $\approx 0.1$ days, implying $\partial \ln \nu_{\mathrm{c}, \mathrm{r}} /$ $\partial \ln t \gtrsim-2$. Thus, the RS break frequencies should be ordered as $\nu_{\mathrm{a}, \mathrm{r}} \lesssim \nu_{\mathrm{m}, \mathrm{r}}<\nu_{\mathrm{c}, \mathrm{r}}$ at $\approx 1.4$ days. This is challenging to achieve with highly relativistic RS models, for which $\nu_{\mathrm{c}, \mathrm{r}} \propto t^{-15 / 8}$ only marginally satisfies the above condition. Upon detailed consideration, no relativistic RS models are able to reproduce the observations, and we focus in the rest of this section on models involving Newtonian or mildly relativistic shocks.

From the energy injection model in Section $5, m \approx 0.35$, implying $q \approx 0.65$ in an ISM environment. For this value of $q$, the RS spectrum evolves as $\frac{\partial \ln \nu_{\mathrm{m}, \mathrm{r}}}{\partial \ln t} \approx-0.66, \frac{\partial \ln \nu_{\mathrm{c}, \mathrm{r}}}{\partial \ln t} \approx-0.68$, and $\frac{\partial \ln F_{\nu, \mathrm{m}, \mathrm{r}}}{\partial \ln t} \approx 0.02$ at $t<t_{\mathrm{E}}$. Thus, the RS peak flux is approximately constant during shock crossing. Evolution after shock crossing depends on the value of $g$.

Under this spectral evolution and the observational constraints described above, we find that an RS model with $g \approx 2.8, t_{\mathrm{E}} \approx 0.25$ days, $\nu_{\mathrm{c}, \mathrm{r}}\left(t_{\mathrm{E}}\right) \approx 1.2 \times 10^{15} \mathrm{~Hz}, \nu_{\mathrm{m}, \mathrm{r}}\left(t_{\mathrm{E}}\right) \approx$ $9.4 \times 10^{10} \mathrm{~Hz}, \quad \nu_{\mathrm{a}, \mathrm{r}}\left(t_{\mathrm{E}}\right) \approx 5.9 \times 10^{10} \mathrm{~Hz}$, and $F_{\nu, \mathrm{m}, \mathrm{r}}\left(t_{\mathrm{E}}\right) \approx$ $22 \mathrm{mJy}$ fits the early optical and X-ray data well (Figure 10). This value of $g$ is intermediate between the values expected for a Newtonian $(g \approx 2.2)$ and relativistic $\mathrm{RS}(g \approx 3)$ for the case of no energy injection. In this model, the X-ray light curve is dominated by the FS at all times, with the suppression prior to 0.25 days arising from energy injection with $m \approx 0.35$. The UV/optical/NIR light curves are dominated by the FS after the end of energy injection at $\approx 0.25$ days and exhibit significant contribution from the RS arising from the injection process prior to this time (Figure 11 ). The radio SEDs at $\approx 1.4,3.4,4.5$, and 8.5 days are well matched by the same RS, propagated to the times of the radio observations (Figure 12). Whereas the model does overpredict the $18-26 \mathrm{GHz}$ observations at 0.5 days, we caution that these frequencies also exhibit the greatest centimeter-band variability before $\approx 8.5$ days, possibly due to extreme interstellar scintillation (ALB18). The large scatter in flux density observed between individual frequencies in the SED further complicates the comparison against the model prediction at this time. Finally, the model also explains the excess in the ALMA light curve ${ }^{37}$ at $\lesssim 3.4$ days (Figure 13).

\subsection{Energy Injection RS-Self-consistency with FS}

If the excess flux density in the early optical and radio observations of GRB 161219B arises from an RS mediating energy injection, we expect the parameters of the RS and FS to be related at the time of cessation of the injection $\left(t_{\mathrm{E}} \approx 0.25\right.$ days). From the FS parameters derived from multiwavelength modeling (Table 8), the Lorentz factor of the FS is $\Gamma\left(t_{\mathrm{E}}\right) \equiv \Gamma_{\mathrm{E}} \approx 22.7$ (Blandford \& McKee 1977). We present a comparison between the observed RS parameters and the values expected by scaling the FS parameters by $\Gamma_{\mathrm{E}}$ in Table 9. We find the peak flux density to match within $5 \%(!)$, suggesting that the RS is not magnetized relative to the FS. The characteristic frequencies also agree upon scaling to within $50 \%$, a stronger match than previously obtained for the Newtonian RS detected in GRB 130427A (Laskar et al. 2013; Perley et al. 2014). Whereas the scaled ratio of the self-absorption frequencies $\nu_{\mathrm{a}, \mathrm{r}} / \Gamma_{\mathrm{E}}^{8 / 5} \nu_{\mathrm{a}, \mathrm{f}}$ is too large by a factor of $\approx 20$, we note that $\nu_{\mathrm{a}, \mathrm{f}}$ is not well constrained, being below the radio band at all times.

The ratio of the cooling frequencies $\nu_{\mathrm{c}, \mathrm{f}} / \nu_{\mathrm{c}, \mathrm{r}} \equiv \mathcal{Q}_{\mathrm{c}, \mathrm{fr}} \approx$ $3.6 \times 10^{2}$ is larger than expected, which is difficult to explain if $R_{\mathrm{B}} \approx 1$. Since $\nu_{\mathrm{c}, \mathrm{r}}$ does not have a strong observational signature being hidden inside the FS spectrum, it could be higher by a factor of several; however, increasing $\nu_{\mathrm{c}, \mathrm{r}}\left(t_{\mathrm{E}}\right)$ beyond $\approx 10^{16} \mathrm{~Hz}$ does begin to affect the X-ray light curve at $10^{-2}$ to $2 \times 10^{-1}$ days. Another possibility is that the Compton $Y$-parameter of the RS is higher than the FS. In the regime $\epsilon_{\mathrm{e}} \gg \epsilon_{\mathrm{B}}$, we expect $Y \approx \sqrt{\eta_{\mathrm{Y}} \epsilon_{\mathrm{e}} / \epsilon_{\mathrm{B}}}$, where $\eta_{\mathrm{Y}}=$ $\left(\nu_{\mathrm{c}} / \nu_{\mathrm{m}}\right)^{-(p-2) / 2}$. Since this ratio is lower than the corresponding ratio for the FS, we expect $Y_{\mathrm{r}} \lesssim Y_{\mathrm{f}}$, and thus inverse Compton cooling cannot explain the observed high ratio of $\nu_{\mathrm{c}, \mathrm{f}}$ to $\nu_{\mathrm{c}, \mathrm{r}}$. The third option is that $\nu_{\mathrm{c}, \mathrm{f}}$ is lower than predicted from the model. A reduced value of $\nu_{\mathrm{c}, \mathrm{f}}$ would require the spectral index above $\nu_{\mathrm{c}, \mathrm{f}}$ to be shallower than $-p / 2$ in order to continue to match the X-ray light curve. Such a flatter spectrum is indeed afforded by the Klein-Nishina correction to the synchrotron

\footnotetext{
37 See Figure 17 in the Appendix for a combined plot showing all 41 observing frequencies.
} 

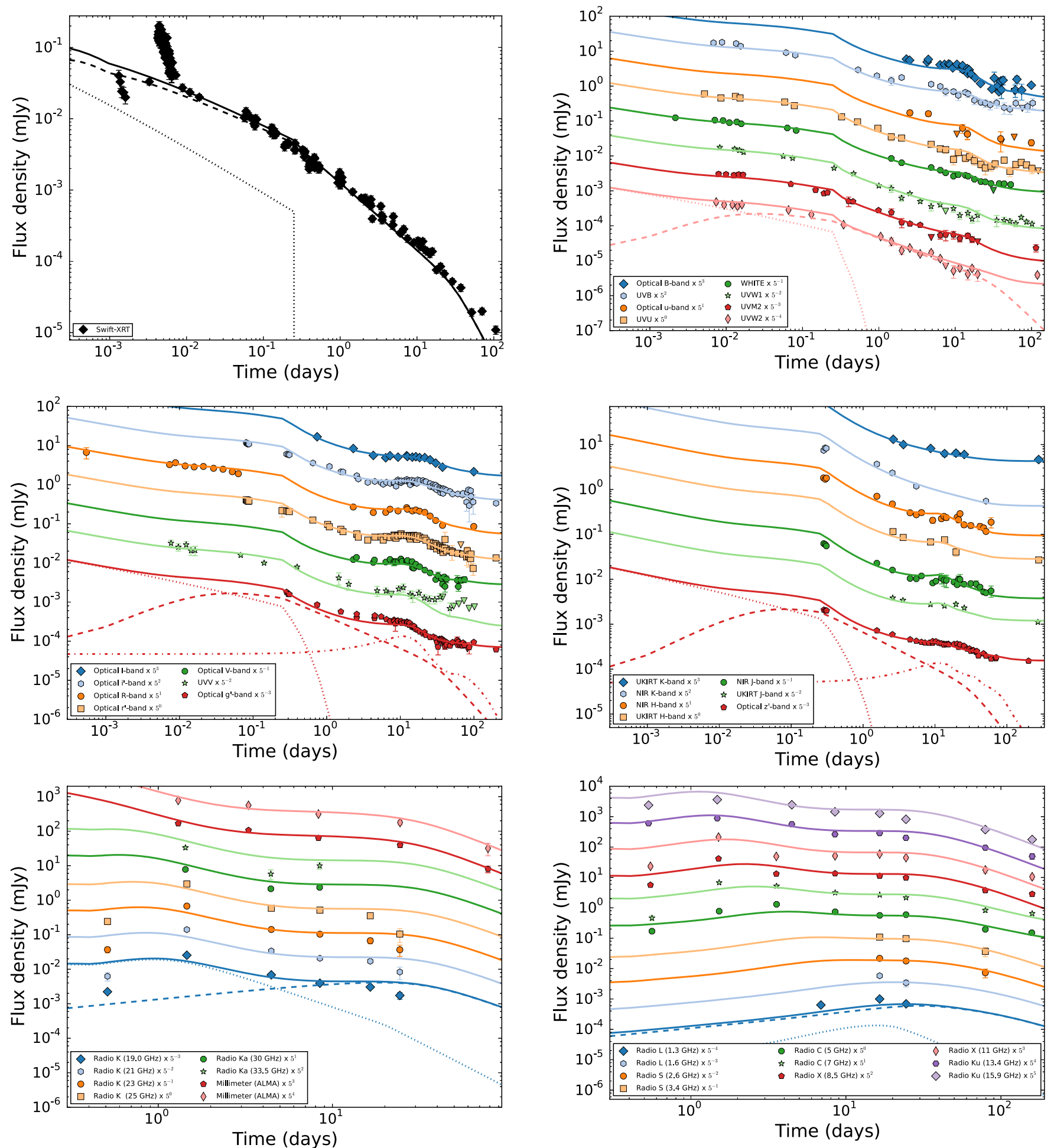

Figure 10. X-ray (top left), UV (top right), optical (center left), NIR (center right), and radio (bottom) light curves of GRB 161219B/SN 2016jca, together with a full FS + RS model with energy injection (solid lines). We show a decomposition of the X-ray, Swift $/ w 2$-band, optical $g^{\prime}$-band, optical $z^{\prime}$-band, $19 \mathrm{GHz}$, and $1.3 \mathrm{GHz}$ light curves into FS (with energy injection; dashed), refreshed RS (dotted), and SN (dotted-dashed) components. The combined model overcomes the deficiencies of the FS-only model (without energy injection; Section 4; Figures 5 and 6) and explains the overall behavior of the light curves at all 41 observing frequencies over 5 orders of magnitude in time. Residual differences in the $10-30 \mathrm{GHz}$ VLA light curves are likely related to the rapid centimeter-band variability observed for this event (Section 3.2). See Figure 17 in the Appendix for a combined plot showing all 41 observing frequencies.

spectrum. We discuss this further in Section 7.5 and the Appendix. We note that recent numerical work suggests that the analytical relations overpredict the RS flux by factors of a few to $\approx 10$ (Nakar \& Piran 2004; Harrison \& Kobayashi 2013); thus, it is possible that the equivalence between these quantities derived above may arise from a coincidence and 


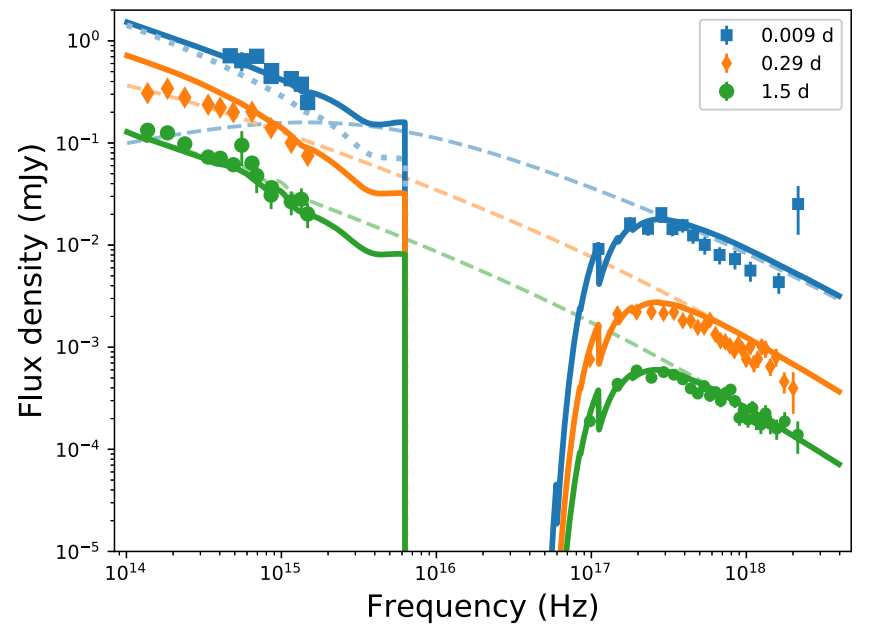

Figure 11. NIR to X-ray SEDs of the afterglow of GRB 161219B at $9 \times 10^{-3}$ days (blue), 0.29 days (orange), and 1.5 days (green), together with the best-fit synchrotron model to the entire multiband data set (Section 4; solid lines). The dip in the UV is the combined effect of extinction in the Galaxy and in the host. The optical data have been interpolated using the average UV light curve (Table 7); the contribution of the host has been removed. The dashed lines represent the FS model without photoelectric absorption or optical extinction; the peak at $\approx 3 \times 10^{15} \mathrm{~Hz}$ in the first epoch is $\nu_{\mathrm{m}}$, and the break at $\approx 2 \times 10^{17} \mathrm{~Hz}$ in the later epochs is $\nu_{\mathrm{c}}$. The SED at $9 \times 10^{-3}$ days is dominated by the RS in the optical (dotted) and the FS in the X-rays (Section 6); RS contribution at later times is negligible in the optical and $\mathrm{X}$-rays. The slight discrepancy in the X-ray SED in the first two epochs may arise from Klein-Nishina corrections to the light curve above $\nu_{\mathrm{c}}$ (Section 7.5).

that the ejecta are magnetized at the level $R_{\mathrm{B}} \approx 5-10$. Since $\nu_{\mathrm{c}, \mathrm{f}} / \nu_{\mathrm{c}, \mathrm{r}} \propto R_{\mathrm{B}}^{3}$ is strongly dependent on $R_{\mathrm{B}}$, even a slight $\mathrm{RS}$ magnetization (say, $R_{\mathrm{B}} \approx 2$, which may be feasible given the uncertainties in the RS parameters) could alleviate the problem. Thus, a combination of a higher value of $\nu_{\mathrm{c}, \mathrm{r}}$, a lower value of $\nu_{\mathrm{c}, \mathrm{f}}$, and slight ejecta magnetization may explain the apparent discrepancy.

The observed strong RS signature in GRB 161219B can be used to place constraints on the circumburst density. The UV/ optical light curves prior to the end of energy injection at $\approx 0.25$ suggest $\nu_{\mathrm{c}, \mathrm{r}} \gtrsim 10^{15} \mathrm{~Hz}$ at this time. Combining Equation (13) with the expression for FS cooling frequency from Granot \& Sari (2002),

$$
\begin{aligned}
\nu_{\mathrm{c}, \mathrm{f}}= & 6.37 \times 10^{13}(p-0.46) e^{-1.16 p}(1+z)^{-1 / 2} \\
& \times \epsilon_{\mathrm{B}}^{-3 / 2} n_{0}^{-1} E_{\mathrm{K}, \text { iso }, 52}^{-1 / 2}\left(1+Y_{\mathrm{f}}\right)^{-2} t_{\mathrm{d}}^{-1 / 2} \mathrm{~Hz} \\
\approx & 5 \times 10^{13} \epsilon_{\mathrm{B},-1}^{-3 / 2} n_{0}^{-1} \mathrm{~Hz} \\
\sim & R_{\mathrm{B}}^{3} \nu_{\mathrm{c}, \mathrm{r}},
\end{aligned}
$$

at the redshift of GRB $161219 \mathrm{~B}$ for $p \approx 2.1, E_{\mathrm{K} \text {,iso }} \approx 5 \times$ $10^{51} \mathrm{erg}, t \approx 0.25$ days, and $Y_{\mathrm{f}} \approx 3$ yields

$$
\begin{aligned}
n_{0} & \approx \frac{5 \times 10^{13} \mathrm{~Hz}}{\nu_{\mathrm{c}, \mathrm{r}}} \epsilon_{\mathrm{B},-1}^{-3 / 2} R_{\mathrm{B}}^{-3} \mathrm{~cm}^{-3} \\
& \lesssim 5 \times 10^{-2} \epsilon_{\mathrm{B},-1}^{-3 / 2} R_{\mathrm{B}}^{-3} \mathrm{~cm}^{-3}
\end{aligned}
$$

For values of $R_{\mathrm{B}} \gtrsim 1$, this constraint becomes stronger. The measured density for GRB 161219B is $n_{0} \approx 4 \times 10^{-4} \mathrm{~cm}^{-3}$, which satisfies this constraint, providing further evidence that bright RS emission is more likely to be detectable in GRBs that occur in low-density environments.

\section{Discussion}

We have presented multiwavelength observations of GRB 161219B and SN 2016jca. The X-ray to radio afterglow can be modeled well as a combination of an FS with energy injection prior to $\approx 0.25$ days and a Newtonian $\mathrm{RS}$ arising from the injection process. The peak frequency and peak flux of the two shocks are fully self-consistent at the deceleration time, indicating low ejecta magnetization. This is the first direct detection of an energy injection RS in a GRB afterglow.

\subsection{Radiative Efficiency}

Comparing the radiated $\gamma$-ray energy with the isotropicequivalent kinetic energy derived from afterglow modeling results in an extremely low radiative efficiency, $\eta_{\text {rad }} \approx 4 \%$. In our previous work on energy injection in GRB afterglows, we found that all events exhibiting a late-time achromatic rebrightening ("extreme rebrightening events," or EREs) in the optical and X-rays also exhibit low efficiencies, ranging from $43 \%$ to as low as 3\% (Laskar et al. 2015). Those events were interpreted in the context of energy injection, with fastmoving ejecta responsible for the $\gamma$-ray emission and slowmoving ejecta carrying the bulk of the kinetic energy.

Extrapolating the energy of the FS in GRB 161219B from $\approx 5 \times 10^{51} \mathrm{erg}$ at 0.25 days to the time of the first optical detection at $\approx 5 \times 10^{-4}$ days, we expect $E_{\mathrm{K} \text {,iso }}\left(t_{\mathrm{X}}\right) \approx 6 \times$ $10^{50} \mathrm{erg}$. Thus, if the energy injection has been carrying on since that time and only the highest Lorentz factor material is responsible for producing the observed $\gamma$-rays, then the required efficiency is higher, $\eta_{\text {rad }} \approx 20 \%$, similar to values obtained in other events (Zhang et al. 2007; Beniamini et al. 2015). The FS Lorentz factor decreases by a factor of $\approx 10$ from $t_{\mathrm{X}}$ to $t_{\mathrm{E}}$. In the framework of the internal shock model, if we associate this with the range of Lorentz factors ejected by the central engine, we expect a theoretical efficiency of $\approx 15 \%$ from internal shocks, comparable to the extrapolated value (Kobayashi et al. 1997). With the caveat that the precise value of the computed efficiency depends on the time at which the injection starts, our observations may provide an independent validation of the internal shock model.

\subsection{Energy Injection and $R S$}

Our observations indicate a relatively slow injection rate, $E \propto t^{0.35}$. If this arises from a distribution of ejecta Lorentz factors, $m \approx 0.35$ corresponds to a shallow ejecta profile, $s \approx 2$. This is similar to the value obtained in GRB 010222 from the X-ray and optical light curves (Björnsson et al. 2002), but lower than the values obtained from multiwavelength modeling of the EREs (Laskar et al. 2015), suggesting that GRB ejecta span a range of Lorentz factor distributions.

We note that the EREs of Laskar et al. (2015) did not exhibit RS signatures, a fact that we suggested may have been due to a gentle injection process. Another explanation could be differences in the ejecta Lorentz factor at the time of the interaction-in the case of GRB 161219B, the RS appears to have been observable from the earliest times in the optical (at $\lesssim 10^{-3}$ days) when the ejecta Lorentz factor is high $(\Gamma \gtrsim 100)$. On the other hand, the onset of injection in the EREs occurred at $\Gamma \sim 20$, which may have been responsible for yielding a fainter RS, or RS emission peaking at frequencies too high to be observable (e.g., in the submillimeter). Since the $\mathrm{RS}$ in GRB 161219B is long-lasting and mildly relativistic, it 

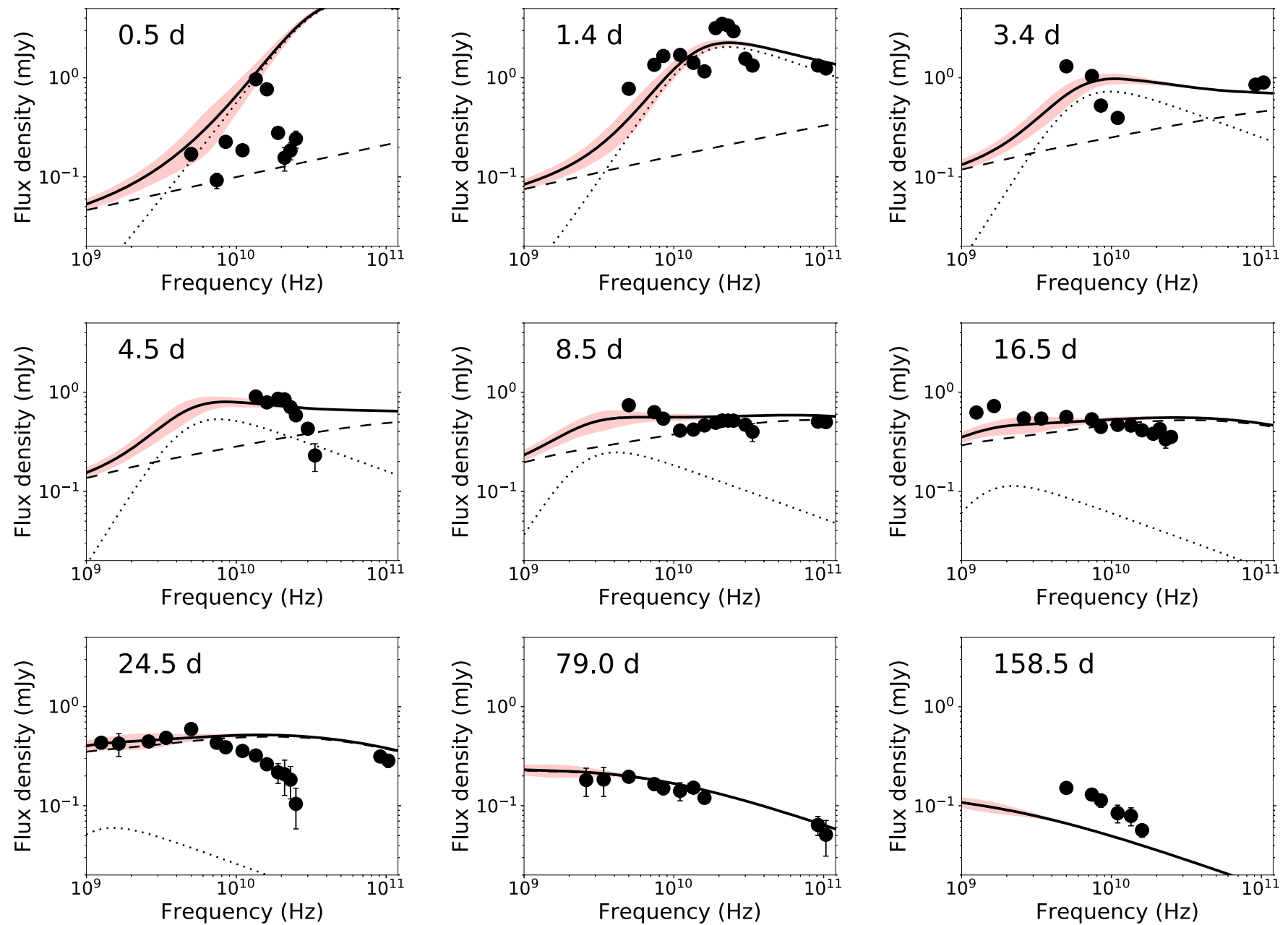

Figure 12. VLA centimeter-band and ALMA millimeter-band SEDs of the afterglow of GRB 161219B at multiple epochs starting at 0.5 days, together with the same FS+RS ISM model as in Figure 10 (solid), decomposed into FS (dashed) and RS (dotted) contributions. The red shaded regions represent the expected variability due to scintillation.

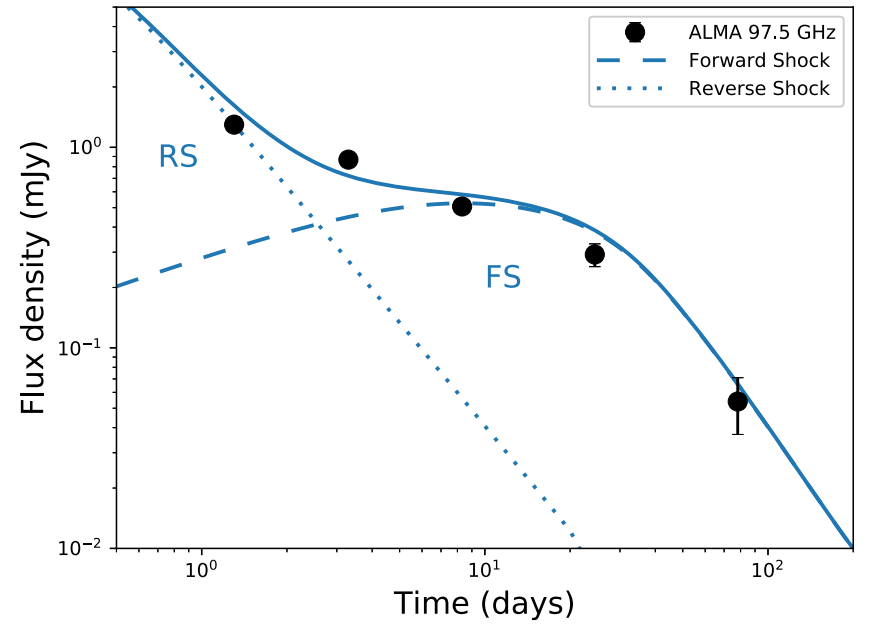

Figure 13. Side-band averaged ALMA $97.5 \mathrm{GHz}$ light curve of GRB 161219B/SN 2016jca, together with the final FS+RS ISM model (solid), decomposed into FS (dashed) and RS (dotted) components. The RS (Section 6) explains the excess in the millimeter band before 3.4 days. The ALMA observations also provide critical constraints on the the FS peak frequency, the peak flux, and the jet break time, as the centimeter-band data exhibit significant variability likely due to extreme scintillation.
Table 9

RS Parameters at $t_{\mathrm{E}}$

\begin{tabular}{ll}
\hline \hline FS (Scaled to $\left.t_{\mathrm{E}}\right)$ & RS \\
\hline$\Gamma_{\mathrm{E}}^{-2} \nu_{\mathrm{m}, \mathrm{f}} \approx 6.2 \times 10^{10} \mathrm{~Hz}$ & $\nu_{\mathrm{m}, \mathrm{r}} \approx 9.4 \times 10^{10} \mathrm{~Hz}$ \\
$\nu_{\mathrm{c}, \mathrm{f}} \approx 4.3 \times 10^{17} \mathrm{~Hz}$ & $\nu_{\mathrm{c}, \mathrm{r}} \approx 1.2 \times 10^{15} \mathrm{~Hz}$ \\
$\Gamma_{\mathrm{E}} F_{\nu, \mathrm{m}, \mathrm{f}} \approx 23 \mathrm{mJy}$ & $F_{\nu, \mathrm{m}, \mathrm{r}} \approx 22 \mathrm{mJy}$ \\
$\Gamma_{\mathrm{E}}^{8 / 3} \nu_{\mathrm{a}, \mathrm{f}} \approx 2.6 \times 10^{9} \mathrm{~Hz}$ & $\nu_{\mathrm{a}, \mathrm{r}} \approx 5.9 \times 10^{10} \mathrm{~Hz}$ \\
\hline
\end{tabular}

continuously decelerates the ejecta; therefore, it is only possible to determine a lower bound on the initial Lorentz factor of the outflow from these observations, $\Gamma_{0} \gtrsim 100$.

The multiwavelength modeling of the EREs was consistent with a constant-density circumburst environment in each case, and the events considered exhibited densities ranging from $10^{-2}$ to $10^{3} \mathrm{~cm}^{-3}$. In contrast, the inferred circumburst density of GRB 161219B is extremely low, $n_{0} \approx 3 \times 10^{-4} \mathrm{~cm}^{-3}$. One possible mechanism for evacuating the environment around massive stars prior to core collapse may be late shell ejections due to super-Eddington winds in the Wolf-Rayet phase or LBV-like eruptions that sweep up the ambient medium; 


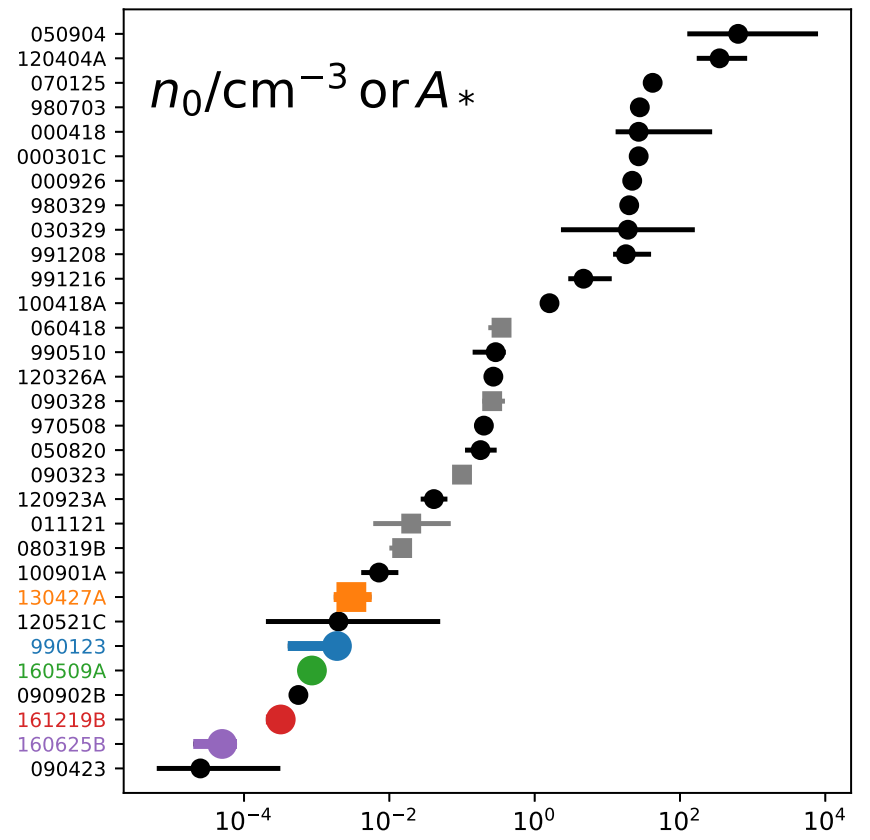

Figure 14. Circumburst density for both ISM (black circles) and wind-like environments (gray squares) for GRBs with multiwavelength observations and modeling (Panaitescu \& Kumar 2002; Yost et al. 2003; Chandra et al. 2008; Cenko et al. 2010, 2011; Laskar et al. 2013, 2014, 2016; Alexander et al. 2017; Tanvir et al. 2017). The GRBs with strong reverse shocks (highlighted as colored symbols) also exhibit some of the lowest circumburst densities of the sample. We note that the remaining three GRBs with the lowest densities (GRB 090423, GRB 090902B, and GRB 120521C) have also been suggested to exhibit RS signatures (Chandra et al. 2010; Cenko et al. 2011; Laskar et al. 2014)

however, the precise degree to which this mechanism is operational and effective for GRB progenitors remains an open question (Weaver et al. 1977; Marston 1997; Moore et al. 2000; Ramirez-Ruiz et al. 2001; Chevalier et al. 2004; Krause et al. 2013; Smith 2014; Margutti et al. 2017). We note that all observed instances of detectable RS emission, without exception, have been in low-density environments ranging from $n_{0} \approx 5 \times 10^{-5}$ to $\approx 10^{-2} \mathrm{~cm}^{-3}$ (Figure 14), and an overwhelming majority of the cases exhibiting strong RS signatures have been detected in constant-density environments (five out of six: GRB 990123, GRB 021211, GRB 041219A, GRB 160509A, and GRB 160625B; Wang et al. 2000; Panaitescu \& Kumar 2002; Kumar \& Panaitescu 2003; Wei 2003; Nakar \& Piran 2004; Fan et al. 2005; Laskar et al. 2013, 2016; Alexander et al. 2017). We have previously speculated that the low-density medium may be responsible for a slow-cooling RS, allowing the RS emission to be detectable for longer (Laskar et al. 2013, 2016; Kopac et al. 2015; Alexander et al. 2017). Indeed, we find a slow-cooling RS in a low-density medium in the case of GRB 161219B also, lending credence to this hypothesis.

\subsection{Comparison with Nearby GRB-SNe and Low- luminosity GRBs}

Owing to the relative faintness of GRB-SNe compared to the afterglow light, only a small fraction (30 out of $\gtrsim 1000$ bursts, or $\lesssim 5 \%$ ) of GRBs have detected SNe. A still smaller number (18) of these have been spectroscopically confirmed to date (Cano et al. 2017b). At the same time, a large fraction of these

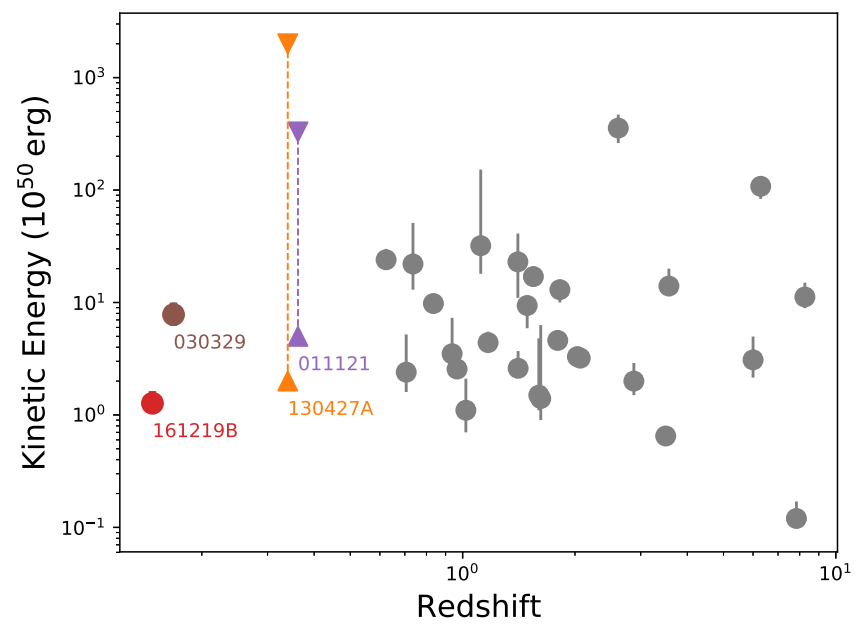

Figure 15. Beaming-corrected kinetic energy of GRB jets as a function of redshift for events with multiwavelength modeling and measured opening angles. We include the SN-associated GRBs GRB 011121 and GRB 130427A with published lower limits on $E_{\mathrm{K}}$; the corresponding upper limits correspond to $E_{\mathrm{K} \text {,iso. }}$.

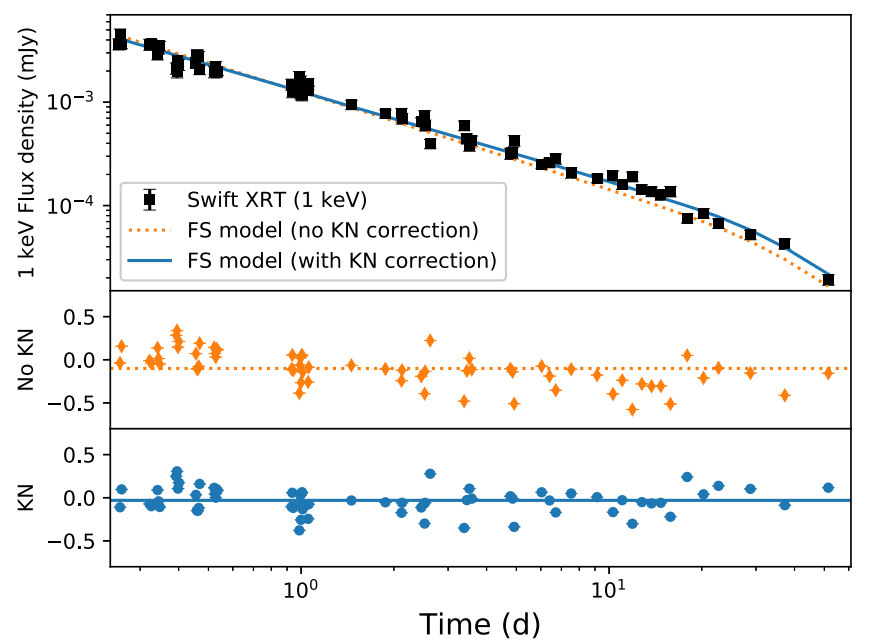

Figure 16. Swift/XRT light curve at $1 \mathrm{keV}$ (top panel) between 0.25 and 51.5 days, together with the FS model described in Section 4 (orange, dotted) and an FS model including KN corrections to the spectrum above $\nu_{\mathrm{c}}$, as well as to the evolution of $\nu_{\mathrm{c}}$ itself (blue, solid). The residuals from the original FS model (middle panel) exhibit systematic trends with time. Including the effects of the KN correction (bottom panel) reduces the trends in the X-ray residuals and yields a better fit to the data. The two models use the same value of $p$.

spectroscopically confirmed GRB-SNe (6/18) appear to have low peak $\gamma$-ray luminosities $\left(L_{\gamma, \text { iso }} \lesssim 10^{48.5} \mathrm{erg} \mathrm{s}^{-1}\right)$, raising the question whether these are representative of the cosmological $(z \gtrsim 1)$ population (Coward 2005; Guetta \& Della Valle 2007; Virgili et al. 2009; Bromberg et al. 2011). In this context, GRB 161219B can be classified as an intermediateluminosity event and is an outlier in the $E_{\gamma \text {,peak }}-E_{\gamma \text {,iso }}$ relation (Amati 2006), together with several low- and intermediateluminosity GRBs (Cano et al. 2017a).

Of the 12 discovered GRB-SNe at $z<0.5$, only one has a measured jet opening angle (GRB 030329; Frail et al. 2005), while two others have lower limits, yielding lower limits on $E_{\mathrm{K}}$ (GRB 011121 and GRB 130427A; Price et al. 2002; Laskar et al. 2013; Perley et al. 2014). Thus, GRB 161219B is the second $\mathrm{SN}$-associated GRB with a well-determined kinetic energy, and it has the lowest kinetic energy of these four events 


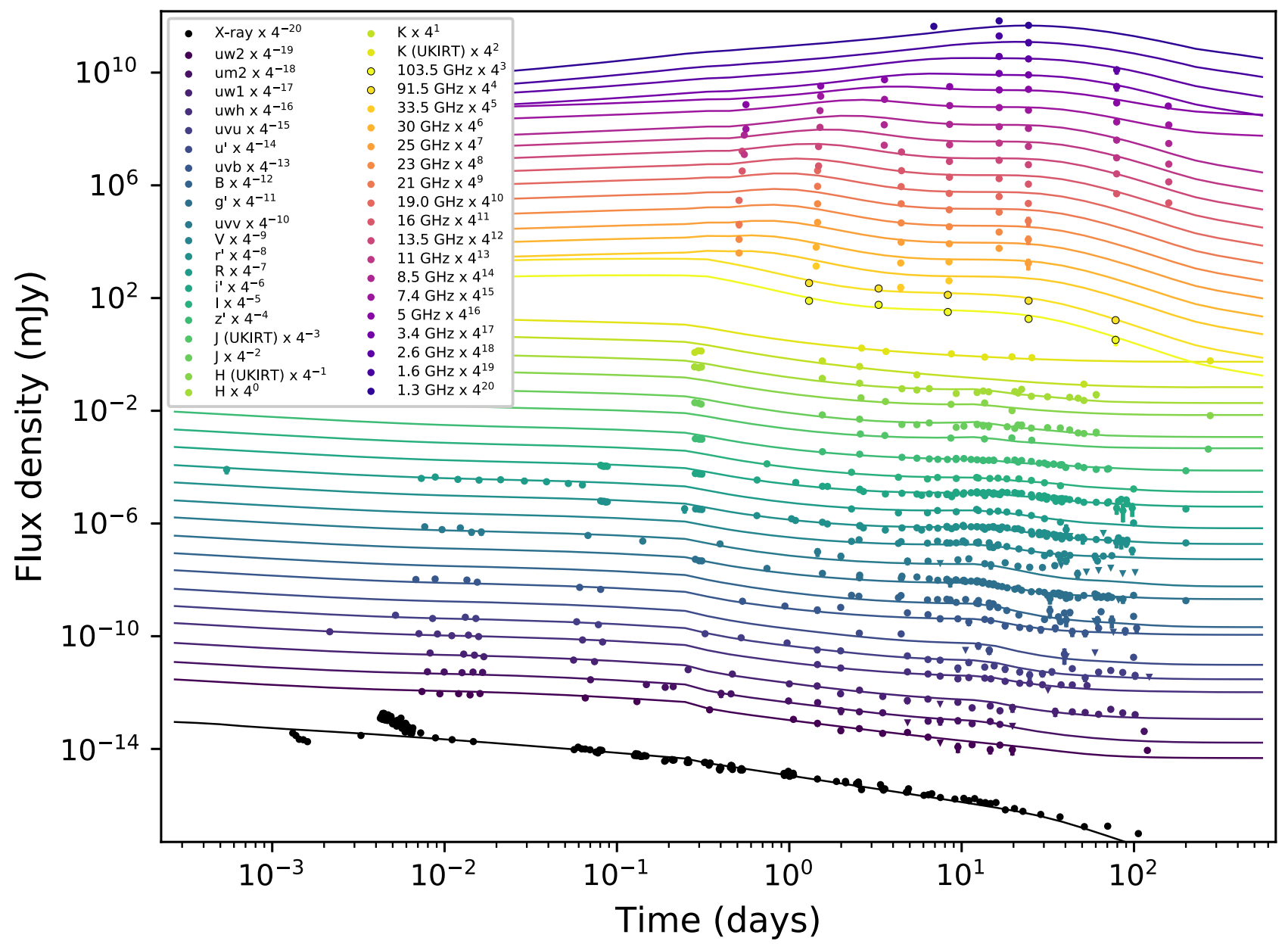

Figure 17. X-ray to radio light curves of GRB 161219B/SN 2016jca, together with the FS+RS model presented in Section 6. The combined model overcomes the deficiencies of the FS-only model (Figures 5 and 6) and explains the overall behavior of the light curves at all 41 observing frequencies over 5 orders of magnitude in time.

(Figure 15). Future observations of GRB-SNe at higher redshifts and at later times to measure their degree of collimation are thus essential for understanding the population of these low-energy events in the context of their cosmological counterparts.

\subsection{High Value of $\epsilon_{\mathrm{e}}$}

The derived value of $\epsilon_{\mathrm{e}} \approx 0.9$ is quite close to 1 , significantly higher than the values of $\epsilon_{\mathrm{e}} \approx 0.1$ derived from simulations of particle acceleration in relativistic shocks (Sironi \& Spitkovsky 2009 , 2011) and also larger than the equipartition value of $\epsilon_{\mathrm{e}} \approx 1 / 3$. Since we expect a nonzero fraction of the shock energy to be transferred to ions, large values of the shock microphysical parameters are problematic. We note here that while the values for $\epsilon_{\mathrm{e}}$ and $\epsilon_{\mathrm{B}}$ given in Table 8 are the best-fit parameters, lower values of $\epsilon_{\mathrm{e}}$ are feasible and are correlated with a lower energy, lower density, and higher value of $\epsilon_{\mathrm{B}}$ (Figure 8). On the other hand, values of $\epsilon_{\mathrm{e}}$ near or above equipartition have been found previously in other works, suggesting that the discrepancy could also arise owing to missing physics in the modeling process (Yost et al. 2003; Cenko et al. 2010, 2011; Laskar et al. 2015, 2016, 2018a). For instance, accounting for a fraction $f \approx 1 \%$ of the electrons accelerated by the shock would reduce $\epsilon_{\mathrm{e}}$ by a corresponding amount (Eichler \& Waxman 2005; Ressler \& Laskar 2017). Unfortunately, the degeneracy in the physical parameters introduced by $f$ precludes a unique determination of this quantity. Relaxing the assumption that all electrons fall into a power-law distribution would also change the definition of $\epsilon_{\mathrm{e}}$ by the factor $(p-2) /(p-1)$ and thus alleviate this discrepancy. The observational signatures of such modifications to the electron energy distribution are under investigation (Ressler \& Laskar 2017; Warren et al. 2017).

\subsection{Unusual X-Ray Properties}

The observed X-ray spectral index of $\beta_{\mathrm{X}}=-0.86 \pm 0.03$ falls between the values $\beta=(1-p) / 2 \approx-0.5$ and $\beta=$ $-p / 2 \approx-1$ for $p \approx 2$, while the $\mathrm{X}$-ray light-curve decline rate also lies between the values expected on either side of the cooling break. Our best-fit model described above requires $\nu_{\mathrm{c}} \approx \nu_{\mathrm{X}}$ for the majority of the $\mathrm{X}$-ray light curve, with $\nu_{\mathrm{c}} \approx 10 \mathrm{keV}$ at $4 \times 10^{-3}$ days and $\nu_{\mathrm{c}} \approx 1 \mathrm{keV}$ at 0.6 days. Since the cooling break is a gentle transition (Granot \& Sari 2002), this may explain the intermediate spectral index and decline rates measured. We note that the hard spectrum above $\nu_{\mathrm{c}}$ may also be the result of Klein-Nishina corrections to the synchrotron spectrum, where we expect a spectral index $\beta_{\mathrm{X}} \approx$ $-3(p-1) / 4 \approx-0.8$ and $\alpha_{\mathrm{X}} \approx-7(p-1) / 8 \approx-0.9$, closer to the observed values (Appendix). With these modifications, the X-ray light curve is modeled much better (Figure 16). Similar effects may explain the slight discrepancy noted between the expected and measured values of $\beta_{\mathrm{X}}$ in the case of GRB 160625B, for which Alexander et al. (2017) also find 
$\nu_{\mathrm{c}} \approx \nu_{\mathrm{X}}$ in an ISM-like environment. A detailed analysis incorporating this effect requires a modified synchrotron spectrum including Klein-Nishina corrections (e.g., Nakar et al. 2009) and is beyond the scope of this work.

\subsection{Radio Excess at 158.5 days}

Whereas the model fits the radio SED at 79 days extremely well, it underpredicts the final radio SED at 158.5 days. The radio light-curve decline rate between these final two epochs steepens from $\alpha=-0.4 \pm 0.2$ at $5 \mathrm{GHz}$ to $-1.1 \pm 0.3$ at $16 \mathrm{GHz}$ but is shallower than the expected value of $\alpha \approx-2$ for $\nu \gtrsim \nu_{\mathrm{m}}$ at $t>t_{\text {jet. }}$. The FS model also underpredicts the last two Swift/XRT observations at $\approx 70.4-106$ days, suggesting that the effect may be panchromatic (Figure 17). Since the Lorentz factor of the FS at these late times is low $(\Gamma \approx 1.2)$, it is possible that electrons at $\gamma \lesssim \gamma_{\min }$ are contributing significantly to the observed radiation, thus invalidating the premise of the radiation model. Another way to achieve a shallower light curve is through a transition to nonrelativistic expansion (Frail et al. 2000; Livio \& Waxman 2000; Sironi \& Giannios 2013); however, this is not expected to occur until $\approx 240$ days (Waxman et al. 1998), while the transition to the deep Newtonian phase takes place even later, near the Sedov time, $t_{\mathrm{ST}} \sim\left(E / \rho c^{5}\right)^{1 / 3} \sim 7 \mathrm{yr}$. Laskar et al. (2018a) found a similar late-time flattening in the centimeter-band light curves of GRB 140311A and considered an early transition to nonrelativistic expansion (such as the FS encountering a density enhancement) as a possible solution. Our model assumes a rapid spreading of the outflow following $t_{\text {jet }}$, whereas recent numerical work suggests that the decollimation process may be more gradual (Zhang \& MacFadyen 2009; van Eerten et al. 2010; van Eerten \& MacFadyen 2012; Duffell \& Laskar 2017). A detailed study of this effect requires numerically calibrated models of the evolution of the synchrotron spectrum during the spreading phase. Analytical calculations in this regime, combined with future late-time $\mathrm{X}$-ray and radio observations of GRB afterglows, will be crucial for clarifying the observed discrepancy. Here we consider the possibility that the flattening is due to the emerging contribution from the underlying host galaxy, and we include an additive constant at these frequencies in the multiband modeling. Further observations of this source at centimeter-band frequencies several years hence would allow distinguishing between these possibilities.

\section{Conclusions}

We have presented detailed multiwavelength observations of GRB 161219B, SN 2016jca, and their host galaxy, including the first ALMA light curve of a GRB afterglow and the first direct detection of an energy injection RS. Through simultaneous multifrequency modeling, we constrain the properties of the afterglow, SN, and host and determine that the GRB occurred in an extremely low density environment, $n_{0} \approx 3 \times 10^{-4} \mathrm{~cm}^{-3}$. The data constrain the beaming angle of the relativistic outflow, allowing us to derive the degree of ejecta collimation $\left(\theta_{\text {jet }} \approx 13^{\circ}\right)$ and to correct the $\gamma$-ray and kinetic energy for beaming, $E_{\gamma} \approx 4.9 \times 10^{48} \mathrm{erg}$ and $E_{\mathrm{K}} \approx$ $1.3 \times 10^{50} \mathrm{erg}$. The prompt efficiency is low, $\eta_{\mathrm{rad}} \approx 4 \%$. The early radio and optical data require an additional emission component, which we interpret as synchrotron radiation arising from a refreshed RS, powered by injection of energy into the
FS through slow-moving ejecta. The combined model explains the X-ray to radio light curves over 8 orders of magnitude in frequency and 5 orders of magnitude in time. We measure a low ejecta magnetization, and our observations provide another confirmation for the internal shock model of GRB prompt emission. The SN component is fainter and evolves faster than SN 1998bw, while the stellar mass of the host galaxy is comparable to that of GRB hosts at $z \lesssim 1$. We conclude that detailed multifrequency radio observations and early optical detections are key to constraining refreshed RSs in GRBs and may yield crucial insight into the production and nature of GRB jets.

We thank the anonymous referee for their helpful comments on improvements of the manuscript. T.L. is a Jansky Fellow of the National Radio Astronomy Observatory. The Berger TimeDomain Group at Harvard is supported in part by the NSF under grant AST-1411763 and by NASA under grant NNX15AE50G. C.G.M. acknowledges support from the Science and Technology Facilities Council. We thank Peter Nugent for generating the SN model. This paper makes use of the following ALMA data: ADS/JAO.ALMA\#2016.1.00819. $\mathrm{T}$ and ADS/JAO.ALMA\#2016.A.00015.S. ALMA is a partnership of ESO (representing its member states), NSF (USA), and NINS (Japan), together with NRC (Canada), NSC and ASIAA (Taiwan), and KASI (Republic of Korea), in cooperation with the Republic of Chile. The Joint ALMA Observatory is operated by ESO, AUI/NRAO, and NAOJ. VLA observations for this study were obtained via project 15A-235. The National Radio Astronomy Observatory is a facility of the National Science Foundation operated under cooperative agreement by Associated Universities, Inc. This work makes use of data supplied by the UK Swift Science Data Centre at the University of Leicester and of data obtained through the High Energy Astrophysics Science Archive Research Center On-line Service, provided by the NASA/ Goddard Space Flight Center. This work includes data obtained with the Swope Telescope at Las Campanas Observatory, Chile, as part of the Swope Time Domain Key Project (PI Piro; co-PIs Shappee, Drout, Madore, Phillips, Foley, and Hsiao). Some of the data presented herein were obtained at the W. M. Keck Observatory, which is operated as a scientific partnership among the California Institute of Technology, the University of California, and the National Aeronautics and Space Administration. The Observatory was made possible by the generous financial support of the W. M. Keck Foundation. This research has made use of the SVO Filter Profile Service, ${ }^{38}$ supported by the Spanish MINECO through grant AyA2014-55216.

\section{Appendix \\ The Klein-Nishina Correction}

The critical energy at which electrons effectively Compton scatter off their own synchrotron photons is given by

$$
\gamma_{\mathrm{self}}=\frac{B_{\mathrm{QED}}}{B},
$$

where $B_{\mathrm{QED}}=4.4 \times 10^{13} \mathrm{G}$ is the quantum critical field and $B$ is the post-shock magnetic field (Nakar et al. 2009). Writing $B=\left(16 \pi \epsilon_{\mathrm{B}} m_{\mathrm{p}} n_{0} c^{2} \Gamma^{2}\right)^{1 / 2}$ for the ISM environment (cgs units) and substituting the relativistic hydrodynamic solution for the

\footnotetext{
${ }^{38}$ http://svo2.cab.inta-csic.es/theory/fps/
} 
Lorentz factor of the FS $(\Gamma)$ as a function of observer time (Blandford \& McKee 1976), we have

$$
\gamma_{\text {self }}=3.5 \times 10^{4} E_{52}^{-1 / 24} n^{-1 / 8} \epsilon_{\mathrm{B}}^{-1 / 6}\left(\frac{t_{\mathrm{d}}}{1+z}\right)^{1 / 8},
$$

where $t_{\mathrm{d}}$ is the observer time in days. For the FS parameters in Table 8, the ordering of the critical Lorentz factors at $\approx 1$ days is $\gamma_{\mathrm{m}} \approx \widehat{\gamma}_{\mathrm{c}}<\gamma_{\text {self }}<\gamma_{\mathrm{c}}<\widehat{\gamma}_{\mathrm{m}}$, where $\gamma^{2} \widehat{\gamma}=\gamma_{\text {self }}^{3}$ (Nakar et al. 2009). Thus, the spectral slope above $\nu_{\mathrm{c}}$ is expected to be $\beta=$ $-3(p-1) / 4 \approx-0.8$ (rather than $\beta=-p / 2 \approx-1.0$ ), agreeing better with the measured X-ray spectral index of $\beta_{\mathrm{X}}=$ $-0.86 \pm 0.03$ at this time. In this regime, we expect $\nu_{\mathrm{c}} \propto t^{-(8-3 p) /(8-2 p)}$ (Nakar et al. 2009). The decline rate of the resulting light curve is, therefore, expected to be marginally shallower: $\alpha \sim 7(1-p) / 8 \approx-0.94$ rather than $\alpha \sim(2-$ $3 p) / 4 \approx-1.05$, in slightly better agreement with the observed decline rate of $\alpha_{\mathrm{X}} \approx-0.82$ during this time. The residual differences may be related to variations in $Y\left(\gamma_{c}\right)$ as the $\mathrm{KN}$ corrected SED transitions between spectral regimes.

\section{ORCID iDs}

Tanmoy Laskar (다 https://orcid.org/0000-0003-1792-2338

Kate D. Alexander (1) https://orcid.org/0000-0002-8297-2473

Edo Berger (10 https://orcid.org/0000-0002-9392-9681

Cristiano Guidorzi (이 https://orcid.org/0000-0001-6869-0835

Raffaella Margutti (i) https://orcid.org/0000-0003-4768-7586

Wen-fai Fong (i) https://orcid.org/0000-0002-7374-935X

Charles D. Kilpatrick (주 https://orcid.org/0000-0002-

5740-7747

Peter Milne (ib https://orcid.org/0000-0002-0370-157X

Shiho Kobayashi (1) https://orcid.org/0000-0001-7946-4200

Ragnhild Lunnan (i) https://orcid.org/0000-0001-9454-4639

Karl M. Menten (i) https://orcid.org/0000-0001-6459-0669

Kunihito Ioka (1) https://orcid.org/0000-0002-3517-1956

Peter K. G. Williams () https://orcid.org/0000-0003-

3734-3587

\section{References}

Akerlof, C., Balsano, R., Barthelmy, S., et al. 1999, Natur, 398, 400 Alexander, K. D., Laskar, T., Berger, E., et al. 2017, ApJ, 848, 69

Alexander, K. D., Laskar, T., Berger, E., et al. 2018, ApJ, submitted (arXiv: 1806.08017)

Aloy, M. A., Müller, E., Ibáñez, J. M., Martí, J. M., \& MacFadyen, A. 2000, ApJL, 531, L119

Amati, L. 2006, MNRAS, 372, 233

Ashall, C., Pian, E., Mazzali, P. A., et al. 2017, arXiv:1702.04339

Baring, M. G., \& Harding, A. K. 1995, AdSpR, 15, 153

Baring, M. G., \& Harding, A. K. 1997, ApJ, 491, 663

Barthelmy, S. D., Barbier, L. M., Cummings, J. R., et al. 2005, SSRv, 120, 143

Beniamini, P., \& Mochkovitch, R. 2017, A\&A, 605, A60

Beniamini, P., Nava, L., Duran, R. B., \& Piran, T. 2015, MNRAS, 454, 1073

Berger, E., Soderberg, A. M., Frail, D. A., \& Kulkarni, S. R. 2003, ApJL, 587, L5

Bhattacharya, D. 2001, BASI, 29, 107

Björnsson, G., Gudmundsson, E. H., \& Jóhannesson, G. 2004, ApJL, 615, L77

Björnsson, G., Hjorth, J., Pedersen, K., \& Fynbo, J. U. 2002, ApJL, 579, L59

Blanchard, P. K., Berger, E., \& Fong, W.-f. 2016, ApJ, 817, 144

Blandford, R. D., \& McKee, C. F. 1976, PhFl, 19, 1130

Blandford, R. D., \& McKee, C. F. 1977, MNRAS, 180, 343

Bloom, J. S., Kulkarni, S. R., \& Djorgovski, S. G. 2002, AJ, 123, 1111

Bromberg, O., Nakar, E., \& Piran, T. 2011, ApJL, 739, L55

Brown, T. M., Baliber, N., Bianco, F. B., et al. 2013, PASP, 125, 1031

Bruzual, G., \& Charlot, S. 2003, MNRAS, 344, 1000
Buckley, D., Potter, S., Kniazev, A., et al. 2016, GCN, 20330 Burrows, D. N., Falcone, A., Chincarini, G., et al. 2007, RSPTA, 365, 1213 Burrows, D. N., Hill, J. E., Nousek, J. A., et al. 2005a, SSRv, 120, 165 Burrows, D. N., Romano, P., Falcone, A., et al. 2005b, Sci, 309, 1833 Cano, Z. 2014, ApJ, 794, 121

Cano, Z., Izzo, L., de Ugarte Postigo, A., et al. 2017a, A\&A, 605, A107 Cano, Z., Wang, S.-Q., Dai, Z.-G., \& Wu, X.-F. 2017b, AdAst, 2017, 8929054 Casali, M., Adamson, A., Alves de Oliveira, C., et al. 2007, A\&A, 467, 777 Cenko, S. B., Frail, D. A., Harrison, F. A., et al. 2010, ApJ, 711, 641 Cenko, S. B., Frail, D. A., Harrison, F. A., et al. 2011, ApJ, 732, 29 Chabrier, G. 2003, PASP, 115, 763

Chandra, P., Cenko, S. B., Frail, D. A., et al. 2008, ApJ, 683, 924 Chandra, P., Frail, D. A., Fox, D., et al. 2010, ApJL, 712, L31

Chevalier, R. A., \& Li, Z.-Y. 2000, ApJ, 536, 195

Chevalier, R. A., Li, Z.-Y., \& Fransson, C. 2004, ApJ, 606, 369

Coward, D. M. 2005, MNRAS, 360, L77

D'Ai, A., Kennea, J. A., Krimm, H. A., et al. 2016, GCN, 20296

Dai, Z. G., \& Cheng, K. S. 2001, ApJL, 558, L109

Dai, Z. G., \& Lu, T. 1998, MNRAS, 298, 87

Dall'Osso, S., Stratta, G., Guetta, D., et al. 2011, A\&A, 526, A121

de Colle, F., Ramirez-Ruiz, E., Granot, J., \& Lopez-Camara, D. 2012, ApJ, 751,57

de Pasquale, M., Kuin, N. P. M., Oates, S., et al. 2015, MNRAS, 449, 1024 de Ugarte Postigo, A., Lundgren, A., Martín, S., et al. 2012, A\&A, 538, A44 Duffell, P. C., \& Laskar, T. 2017, ApJ, submitted (arXiv:17100.7253)

Duffell, P. C., \& MacFadyen, A. I. 2015, ApJ, 806, 205

Eichler, D., \& Granot, J. 2006, ApJL, 641, L5

Eichler, D., \& Waxman, E. 2005, ApJ, 627, 861

Evans, P. A., Beardmore, A. P., Page, K. L., et al. 2007, A\&A, 469, 379

Evans, P. A., Beardmore, A. P., Page, K. L., et al. 2009, MNRAS, 397, 1177

Fan, Y. Z., Zhang, B., \& Wei, D. M. 2005, ApJL, 628, L25

Fenimore, E. E., Epstein, R. I., \& Ho, C. 1993, A\&AS, 97, 59

Foreman-Mackey, D., Hogg, D. W., Lang, D., \& Goodman, J. 2013, PASP, 125,306

Frail, D. A., Soderberg, A. M., Kulkarni, S. R., et al. 2005, ApJ, 619, 994

Frail, D. A., Waxman, E., \& Kulkarni, S. R. 2000, ApJ, 537, 191

Frederiks, D., Golenetskii, S., Aptekar, R., et al. 2016, GCN, 20323

Fruchter, A. S., Levan, A. J., Strolger, L., et al. 2006, Natur, 441, 463

Gao, H., Lei, W.-H., Zou, Y.-C., Wu, X.-F., \& Zhang, B. 2013, NewAR, 57,141

Gehrels, N., Chincarini, G., Giommi, P., et al. 2004, ApJ, 611, 1005

Genet, F., Daigne, F., \& Mochkovitch, R. 2007, MNRAS, 381, 732

Ghisellini, G., Nardini, M., Ghirlanda, G., \& Celotti, A. 2009, MNRAS, 393,253

Giannios, D., Mimica, P., \& Aloy, M. A. 2008, A\&A, 478, 747

Granot, J., Königl, A., \& Piran, T. 2006, MNRAS, 370, 1946

Granot, J., \& Kumar, P. 2006, MNRAS, 366, L13

Granot, J., Nakar, E., \& Piran, T. 2003, Natur, 426, 138

Granot, J., Panaitescu, A., Kumar, P., \& Woosley, S. E. 2002, ApJL, 570, L61 Granot, J., \& Piran, T. 2012, MNRAS, 421, 570

Granot, J., \& Sari, R. 2002, ApJ, 568, 820

Greiner, J., Krühler, T., Nardini, M., et al. 2013, A\&A, 560, A70

Guetta, D., \& Della Valle, M. 2007, ApJL, 657, L73

Guidorzi, C., Vergani, S. D., Sazonov, S., et al. 2007, A\&A, 474, 793

Hamly, N. C., Collins, R. S., Cross, N. J. G., et al. 2008, MNRAS, 384, 637

Harrison, R., \& Kobayashi, S. 2013, ApJ, 772, 101

Henden, A. A., Templeton, M., Terrell, D., et al. 2016, yCat, 2336

Holland, S. T., De Pasquale, M., Mao, J., et al. 2012, ApJ, 745, 41

Huang, Y. F., Cheng, K. S., \& Gao, T. T. 2006, ApJ, 637, 873

Ioka, K., Kobayashi, S., \& Zhang, B. 2005, ApJ, 631, 429

Jeffreys, H. 1946, RSPTA, 186, 453

Jin, Z. P., Yan, T., Fan, Y. Z., \& Wei, D. M. 2007, ApJL, 656, L57

Jóhannesson, G., Björnsson, G., \& Gudmundsson, E. H. 2006, ApJ, 647, 1238

Kilpatrick, C. D., Foley, R. J., Drout, M. R., et al. 2018, MNRAS, 473, 4805 Kobayashi, S. 2000, ApJ, 545, 807

Kobayashi, S., Piran, T., \& Sari, R. 1997, ApJ, 490, 92

Kobayashi, S., \& Sari, R. 2000, ApJ, 542, 819

Kobayashi, S., \& Zhang, B. 2003, ApJL, 582, L75

Kobayashi, S., \& Zhang, B. 2007, ApJ, 655, 973

Kong, S. W., Wong, A. Y. L., Huang, Y. F., \& Cheng, K. S. 2010, MNRAS, 402, 409

Kopac, D., Mundell, C. G., Kobayashi, S., et al. 2015, ApJ, 806, 179

Krause, M., Fierlinger, K., Diehl, R., et al. 2013, A\&A, 550, A49

Kriek, M., van Dokkum, P. G., Labbé, I., et al. 2009, ApJ, 700, 221

Krolik, J. H., \& Pier, E. A. 1991, ApJ, 373, 277

Kulkarni, S. R., Frail, D. A., Sari, R., et al. 1999, ApJL, 522, L97 
Kumar, P., \& Panaitescu, A. 2000, ApJL, 541, L51

Kumar, P., \& Panaitescu, A. 2003, MNRAS, 346, 905

Kumar, P., \& Piran, T. 2000, ApJ, 532, 286

Laskar, T., Alexander, K. D., Berger, E., et al. 2016, ApJ, 833, 88

Laskar, T., Berger, E., Chornock, R., et al. 2018a, ApJ, 858, 68

Laskar, T., Berger, E., Margutti, R., et al. 2015, ApJ, 814, 1

Laskar, T., Berger, E., Margutti, R., et al. 2018b, ApJ, 859, 134

Laskar, T., Berger, E., Tanvir, N., et al. 2014, ApJ, 781, 1

Laskar, T., Berger, E., Zauderer, B. A., et al. 2013, ApJ, 776, 119

Levan, A., Nugent, P., Fruchter, A., et al. 2005, ApJ, 624, 880

Levesque, E. M., Kewley, L. J., Berger, E., \& Jabran Zahid, H. 2010, AJ, 140,1557

Li, L., Liang, E.-W., Tang, Q.-W., et al. 2012, ApJ, 758, 27

Lithwick, Y., \& Sari, R. 2001, ApJ, 555, 540

Livio, M., \& Waxman, E. 2000, ApJ, 538, 187

Lyutikov, M., \& Camilo Jaramillo, J. 2017, ApJ, 835, 206

MacFadyen, A. I., \& Woosley, S. E. 1999, ApJ, 524, 262

Mangano, V., Holland, S. T., Malesani, D., et al. 2007, A\&A, 470, 105

Margutti, R., Bernardini, G., Barniol Duran, R., et al. 2011, MNRAS, 410, 1064

Margutti, R., Genet, F., Granot, J., et al. 2010a, MNRAS, 402, 46

Margutti, R., Guidorzi, C., Chincarini, G., et al. 2010b, MNRAS, 406, 2149

Margutti, R., Kamble, A., Milisavljevic, D., et al. 2017, ApJ, 835, 140

Marshall, F. E., \& D'Ai, A. 2016, GCN, 20306

Marston, A. P. 1997, ApJ, 475, 188

Mazaeva, E., Mokhnatkin, A., Pozanenko, A., Volnova, A., \& Molotov, I. 2016, GCN, 20309

McMullin, J. P., Waters, B., Schiebel, D., Young, W., \& Golap, K. 2007, in ASP Conf. Ser. 376, Astronomical Data Analysis Software and Systems XVI, ed. R. A. Shaw, F. Hill, \& D. J. Bell (San Francisco, CA: ASP), 127

Melandri, A., Guidorzi, C., Kobayashi, S., et al. 2009, MNRAS, 395, 1941

Melandri, A., Mundell, C. G., Kobayashi, S., et al. 2008, ApJ, 686, 1209

Metzger, B. D., Giannios, D., Thompson, T. A., Bucciantini, N., \& Quataert, E. 2011, MNRAS, 413, 2031

Moore, B. D., Hester, J. J., \& Scowen, P. A. 2000, AJ, 119, 2991

Nakar, E., Ando, S., \& Sari, R. 2009, ApJ, 703, 675

Nakar, E., \& Piran, T. 2003, ApJ, 598, 400

Nakar, E., \& Piran, T. 2004, MNRAS, 353, 647

Narayan, R., Piran, T., \& Kumar, P. 2001, ApJ, 557, 949

Nardini, M., Elliott, J., Filgas, R., et al. 2014, A\&A, 562, A29

Nardini, M., Ghisellini, G., Ghirlanda, G., \& Celotti, A. 2010, MNRAS, 403,1131

Nayana, A. J., \& Chandra, P. 2016, GCN, 20344

Nousek, J. A., Kouveliotou, C., Grupe, D., et al. 2006, ApJ, 642, 389

Oke, J. B., Cohen, J. G., Carr, M., et al. 1995, PASP, 107, 375

Palmer, D. M., Barthelmy, S. D., Cummings, J. R., et al. 2016, GCN, 20308

Panaitescu, A., \& Kumar, P. 2002, ApJ, 571, 779

Panaitescu, A., \& Kumar, P. 2004, MNRAS, 350, 213

Panaitescu, A., Mészáros, P., Gehrels, N., Burrows, D., \& Nousek, J. 2006, MNRAS, 366, 1357

Panaitescu, A., Vestrand, W. T., \& Wózniak, P. 2013, MNRAS, 433, 759

Patat, F., Cappellaro, E., Danziger, J., et al. 2001, ApJ, 555, 900

Pei, Y. C. 1992, ApJ, 395, 130

Perley, D. A., Cenko, S. B., Corsi, A., et al. 2014, ApJ, 781, 37

Piran, T. 2005, RvMPh, 76, 1143

Price, P. A., Berger, E., Reichart, D. E., et al. 2002, ApJL, 572, L51

Ramirez-Ruiz, E., Dray, L. M., Madau, P., \& Tout, C. A. 2001, MNRAS, 327,829

Rees, M. J., \& Meszaros, P. 1992, MNRAS, 258, 41P

Rees, M. J., \& Meszaros, P. 1998, ApJL, 496, L1

Ressler, S. M., \& Laskar, T. 2017, ApJ, 845, 150
Rest, A., Stubbs, C., Becker, A. C., et al. 2005, ApJ, 634, 1103

Rhoads, J. E. 1999, ApJ, 525, 737

Rickett, B. J. 1990, ARA\&A, 28, 561

Roming, P. W. A., Kennedy, T. E., Mason, K. O., et al. 2005, SSRv, 120, 95

Sari, R., \& Esin, A. A. 2001, ApJ, 548, 787

Sari, R., \& Mészáros, P. 2000, ApJL, 535, L33

Sari, R., \& Piran, T. 1999a, ApJL, 517, L109

Sari, R., \& Piran, T. 1999b, ApJ, 520, 641

Sari, R., Piran, T., \& Halpern, J. P. 1999, ApJL, 519, L17

Sari, R., Piran, T., \& Narayan, R. 1998, ApJL, 497, L17

Schlafly, E. F., \& Finkbeiner, D. P. 2011, ApJ, 737, 103

Scolnic, D., Casertano, S., Riess, A., et al. 2015, ApJ, 815, 117

Shao, L., \& Dai, Z. G. 2007, ApJ, 660, 1319

Sironi, L., \& Giannios, D. 2013, ApJ, 778, 107

Sironi, L., \& Spitkovsky, A. 2009, ApJ, 698, 1523

Sironi, L., \& Spitkovsky, A. 2011, ApJ, 726, 75

Smith, N. 2014, ARA\&A, 52, 487

Soderberg, A., \& Ramirez-Ruiz, E. 2003, MNRAS, 345, 854

Soderberg, A. M., \& Ramirez-Ruiz, E. 2002, MNRAS, 330, L24

Steele, I. A., Smith, R. J., Rees, P. C., et al. 2004, Proc. SPIE, 5489, 679

Svensson, K. M., Levan, A. J., Tanvir, N. R., Fruchter, A. S., \& Strolger, L.-G. 2010, MNRAS, 405, 57

Tanvir, N. R., Kruehler, T., Wiersema, K., et al. 2016, GCN, 20321

Tanvir, N. R., Laskar, T., Levan, A. J., et al. 2017, arXiv:1703.09052

Tchekhovskoy, A., McKinney, J. C., \& Narayan, R. 2008, MNRAS, 388, 551

Thompson, A. R., Moran, J. M., \& Swenson, G. W., Jr. 2001, Interferometry and Synthesis in Radio Astronomy (2nd ed.; New York: Wiley)

Toma, K., Ioka, K., Yamazaki, R., \& Nakamura, T. 2006, ApJL, 640, L139

Troja, E., Sakamoto, T., Guidorzi, C., et al. 2012, ApJ, 761, 50

Uhm, Z. L. 2011, ApJ, 733, 86

Uhm, Z. L., \& Zhang, B. 2014, ApJ, 789, 39

Uhm, Z. L., Zhang, B., Hascoët, R., et al. 2012, ApJ, 761, 147

van der Horst, A. J., Paragi, Z., de Bruyn, A. G., et al. 2014, MNRAS, 444,3151

van Eerten, H., Zhang, W., \& MacFadyen, A. 2010, ApJ, 722, 235

van Eerten, H. J., \& MacFadyen, A. I. 2012, ApJ, 751, 155

Virgili, F. J., Liang, E.-W., \& Zhang, B. 2009, MNRAS, 392, 91

Virgili, F. J., Mundell, C. G., Pal'shin, V., et al. 2013, ApJ, 778, 54

Wang, X. Y., Dai, Z. G., \& Lu, T. 2000, MNRAS, 319, 1159

Warren, D. C., Ellison, D. C., Barkov, M. V., \& Nagataki, S. 2017, ApJ, 835,248

Waxman, E., Kulkarni, S. R., \& Frail, D. A. 1998, ApJ, 497, 288

Weaver, R., McCray, R., Castor, J., Shapiro, P., \& Moore, R. 1977, ApJ, 218,377

Wei, D. M. 2003, A\&A, 402, L9

Willingale, R., Starling, R. L. C., Beardmore, A. P., Tanvir, N. R., \& O'Brien, P. T. 2013, MNRAS, 431, 394

Woods, E., \& Loeb, A. 1995, ApJ, 453, 583

Woosley, S. E. 1993, ApJ, 405, 273

Woosley, S. E., \& Bloom, J. S. 2006, ARA\&A, 44, 507

Wu, X. F., Dai, Z. G., Huang, Y. F., \& Lu, T. 2003, MNRAS, 342, 1131

Yost, S. A., Harrison, F. A., Sari, R., \& Frail, D. A. 2003, ApJ, 597, 459

Zhang, B., Fan, Y. Z., Dyks, J., et al. 2006, ApJ, 642, 354

Zhang, B., Kobayashi, S., \& Mészáros, P. 2003, ApJ, 595, 950

Zhang, B., Liang, E., Page, K. L., et al. 2007, ApJ, 655, 989

Zhang, B., \& Mészáros, P. 2001, ApJL, 552, L35

Zhang, B., \& Mészáros, P. 2002, ApJ, 566, 712

Zhang, W., \& MacFadyen, A. 2009, ApJ, 698, 1261

Zhang, W., Woosley, S. E., \& MacFadyen, A. I. 2003, ApJ, 586, 356

Zou, Y. C., Wu, X. F., \& Dai, Z. G. 2005, MNRAS, 363, 93 